
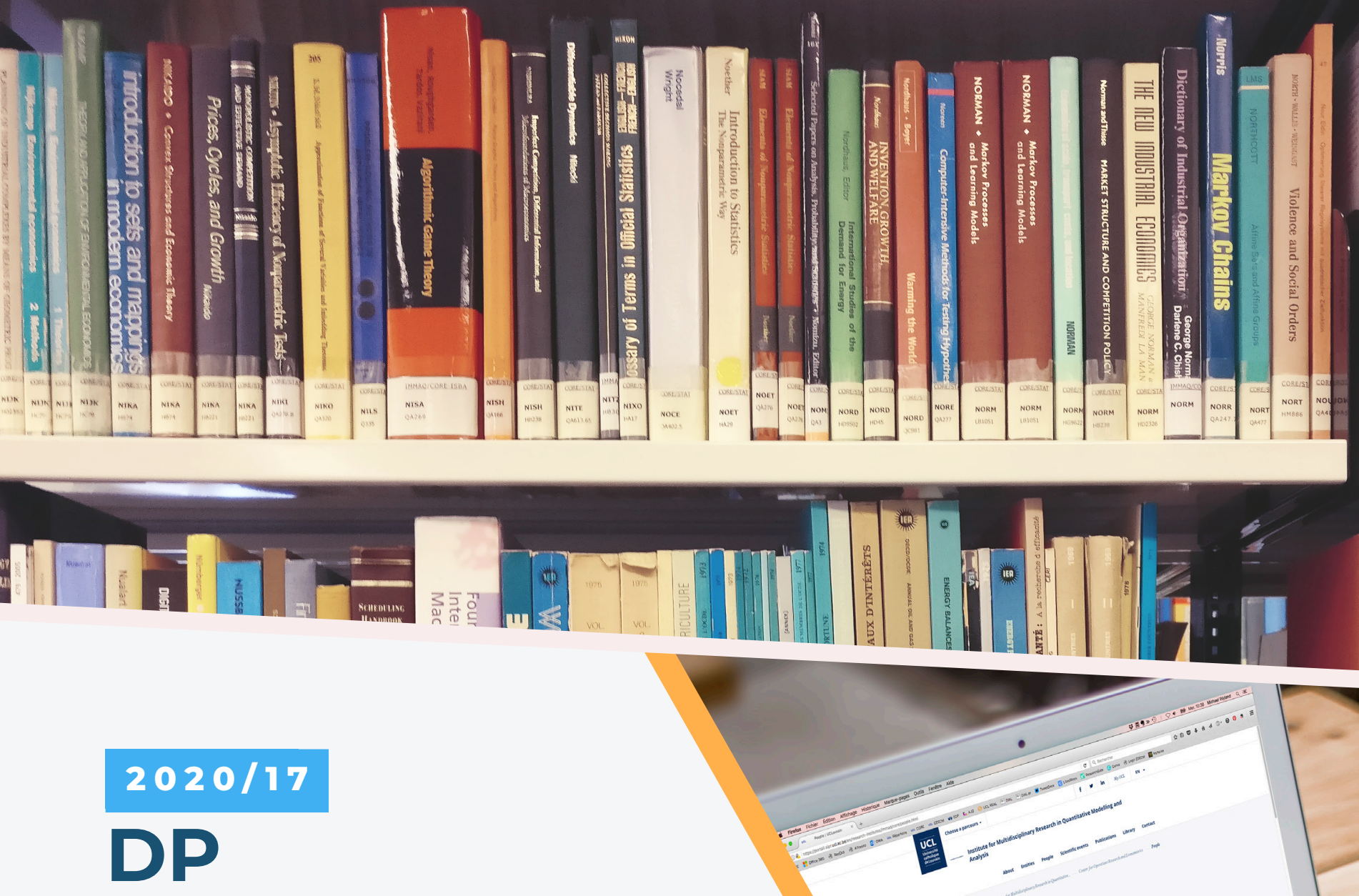

Chenghong Luo, Ana Mauleon and Vincent Vannetelbosch

Segregation versus assimilation in friendship networks with farsighted and myopic agents 


\section{CORE}

Voie du Roman Pays 34, L1.03.01

B-1348 Louvain-la-Neuve

Tel (32 10) 474304

Email: immaq-library@uclouvain.be

https://uclouvain.be/en/research-institutes/

lidam/core/discussion-papers.html 


\title{
Segregation versus Assimilation in Friendship Networks with Farsighted and Myopic Agents
}

\author{
Chenghong Luo* Ana Mauleon ${ }^{\dagger} \quad$ Vincent Vannetelbosch ${ }^{\ddagger}$
}

May 12, 2020

\begin{abstract}
We reconsider de Marti and Zenou (2017) model of friendship network formation where individuals belong to two different communities. Benefits from direct and indirect connections decay with distance while costs of forming links depend on community memberships. Individuals are now either farsighted or myopic when deciding about the friendship links they want to form. When all individuals are myopic many inefficient friendship networks (e.g. complete segregation) can arise. When the larger (smaller) community is farsighted while the smaller (larger) community is myopic, the friendship network where the myopic community is assimilated into the farsighted community is the unique stable network when inter-community costs are large. In fact, farsightedness helps the society to avoid ending up segregated. Once inter-community costs are small enough, the complete integration network become stable. Finally, when all individuals are farsighted, the friendship network where the smaller community ends up being assimilated into the dominant community is likely to arise.
\end{abstract}

Key words: friendship networks; stable sets; myopic and farsighted players; assimilation; segregation.

JEL Classification: A14, C70, D20.

${ }^{*}$ CORE, Université catholique de Louvain, Louvain-la-Neuve, Belgium; Ca' Foscari University of Venice, Venice, Italy. E-mail: chenghong.luo@uclouvain.be

${ }^{\dagger}$ CEREC, Université Saint-Louis - Brussels; CORE, Université catholique de Louvain, Louvain-laNeuve, Belgium. E-mail: ana.mauleon@usaintlouis.be

$\ddagger$ CORE, Université catholique de Louvain, Louvain-la-Neuve, Belgium. E-mail: vincent.vannetelbosch@uclouvain.be 


\section{Introduction}

Social networks or friendship networks are important in obtaining information on goods and services, like product information or information about job opportunities. Individuals are often regrouped into communities based on their ethnicity, religion, income, education, etc. (see e.g. de Marti and Zenou, 2017; Patacchini and Zenou, 2016). Beside belonging to different communities, individuals often differ in their degree of farsightedness, i.e., their ability to forecast how others will react to the decisions they take. Indeed, recent experiments on network formation provide evidence in favor of a mixed population consisting of both myopic and (limited) farsighted individuals (see Kirchsteiger, Mantovani, Mauleon and Vannetelbosch, 2016; Teteryatnikova and Tremewan, 2020). The degree of farsightedness or the depth of reasoning are likely to be correlated with other relevant attributes such as education, income, age, etc. (see Mauersberger and Nagel, 2018).

The aim of this paper is to provide a theoretical study of how different degrees of farsightedness will affect the formation of friendship relationships when individuals can belong to various communities. ${ }^{1}$ In particular, we are interested in addressing the following set of questions. What are the friendship network structures that may endogenously arise once individuals belonging to two different communities can be either myopic or farsighted in forming links? When do we observe integration, segregation or (partial) assimilation? Does farsightedness help to bridge communities and to more integrated societies? Are farsighted individuals more likely to be linked to others who have different characteristics? How might the network structure change if the dominant community is farsighted while the other one is myopic? Do myopic individuals end up assimilated to the dominant community? Are individual incentives to link adequate from a social welfare point of view? Does it improve efficiency if some individuals become farsighted? And if yes, whom?

To answer these questions we reconsider de Marti and Zenou (2017) model of network formation where individuals belong to two different communities. Communities may be defined along social categories such as ethnicity, religion, education, income, etc. In contrast to de Marti and Zenou (2017) where all individuals were myopic, we now allow the possibility of having a mixed population composed of both myopic and farsighted individuals. Myopic or farsighted individuals decide with whom they want to form a link, according to a utility function that weights the costs and benefits of each connection. Farsighted individuals are able to anticipate that once they add or delete some links, other individuals could add or delete links afterwards. Benefits of a friendship connection decrease with distance in the network, while the cost of a link depends on the type of individuals involved. Two individuals from the same community face a low linking cost,

\footnotetext{
${ }^{1}$ Jackson (2008) and Goyal (2007) provide a comprehensive introduction to the theory of social and economic networks. Mauleon and Vannetelbosch (2016) give an overview of the solution concepts for solving network formation games. In Bramoullé, Galeotti and Rogers (2016), one can find the recent developments on the economics of networks.
} 
while the cost of forming a friendship relationship between two individuals from different communities decreases with the rate of exposure of each of them to the other community.

We adopt the notion of myopic-farsighted stable set to determine the friendship networks that emerge when some individuals are myopic while others are farsighted. ${ }^{2}$ A myopic-farsighted stable set is the set of networks satisfying internal and external stability with respect to the notion of myopic-farsighted improving path. That is, a set of networks is a myopic- farsighted stable set if there is no myopic-farsighted improving path between networks within the set and there is a myopic-farsighted improving path from any network outside the set to some network within the set. A myopic-farsighted improving path is simply a sequence of networks that can emerge when farsighted individuals form or delete links based on the improvement the end network offers relative to the current network while myopic individuals form or delete links based on the improvement the resulting network offers relative to the current network.

When all individuals are myopic, de Marti and Zenou (2017) already show that many friendship networks can be stable. In the case of low intra-community costs, the complete integration is stable when inter-community costs are sufficiently low. For higher intercommunity costs, the complete segregation becomes stable. They also point out that some asymmetric network configurations can be stable. For instance, the network in which both communities are fully intra-connected and where there is only one bridge link can be stabilized. In addition, we show that friendship networks where one community is fully or partially assimilated to the other community can also emerge in the long run.

What happens when the population is composed of both myopic and farsighted individuals? Suppose first that all members of one community are farsighted while all members of the other community are myopic. We show that, in the case of low intra-community costs, there is a single friendship network that emerges in the long run when inter-community costs are large enough: the friendship network where the myopic community ends up being assimilated into the farsighted community. Precisely, a singleton set consisting of the network where the myopic community is assimilated into the farsighted community is the unique myopic-farsighted stable set. Farsighted individuals are able destabilize the complete segregated network by luring the myopic individuals with the prospect of forming a friendship network where the farsighted community is fully assimilated into the myopic community. From such friendship network, farsighted individuals are able to induce a switch towards the opposite fully assimilated network, the friendship network where the myopic community is fully assimilated into the farsighted community, where they achieve their best outcome. When inter-community costs are smaller, the complete integration network becomes again stable whatever the number of farsighted and myopic individuals within the population.

\footnotetext{
${ }^{2}$ Herings, Mauleon and Vannetelbosch (2020) were first to define the myopic-farsighted stable set for two-sided matching problems. This notion is extended to R\&D network formation with pairwise deviations in Mauleon, Sempere-Monerris and Vannetelbosch (2020) and to general network formation problems in Luo, Mauleon and Vannetelbosch (2020).
} 
One may wonder if assimilated friendship networks are still stable once individuals from the myopic community become farsighted. We find that, when all the population is farsighted and intra-community costs are low, the friendship network where the smaller community is fully assimilated into the larger or dominant community is likely to emerge in the long run whatever the inter-community costs. However, the opposite fully assimilated network and the complete segregation network are very unlikely to arise. In addition, the complete segregation network is even Pareto-dominated by the friendship network where the smaller community is fully assimilated into the dominant community. In fact, in terms of efficiency, either the complete integration network or the network where the smaller community is fully assimilated into the dominant one are the optimal network structures when intra-community costs are low. Thus, for recovering efficiency, it is better to make individuals belonging to the dominant community farsighted instead of individuals of the smaller community.

In the case of intermediate intra-community costs, many friendship networks are again stable when all individuals are myopic. However, we show that if there are enough farsighted individuals, independently to which community they belong, then a star network with a myopic in the center will arise. In addition, star networks turn to be the efficient networks for intermediate intra-community costs. Hence, a mixed population of farsighted and myopic individuals solve the tension between stability and efficiency.

We now turn to the related literature. There is an extensive literature using network models to explain the fact that individuals are more likely to be linked to individuals who have similar characteristics. Currarini, Jackson and Pin (2009) develop a dynamic random matching model with a population formed by groups of different sizes and show that segregation in social networks results from the decisions of the individuals involved and/or from the ways in which individuals meet and interact. In equilibrium, individuals' behavior is totally homogeneous within the same group of individuals. Bramoullé, Currarini, Jackson, Pin and Rogers (2012) develop a model of dynamic matching with both random meetings and network-based search. They show that majority and minority groups have different patterns of interactions and that relative homophily in the network is strongest when groups have equal size, and vanishes as groups have increasingly unequal sizes. ${ }^{3}$

Despite strong empirical evidence, few models of network formation with differentiated communities have studied the impact of social networks on the long-run integration outcome of minorities. Jackson and Rogers (2005) extend the Jackson and Wolinsky (1996)'s connection model by including two communities and assuming that the cost of linking two individuals from different communities is exogenous and independent of the behavior of the two individuals involved in the link. Johnson and Gilles (2000) add a geographical

\footnotetext{
${ }^{3}$ Mele (2017) proposes a dynamic model of network formation that combines strategic and random networks features. In each period an individual meets another individual and decides whether to form a new link, keep an existing link or do nothing. He shows that the model converges to a unique stationary equilibrium distribution over networks.
} 
dimension to Jackson and Wolinsky (1996)'s connection model assuming that the cost of a link is proportional to the geographical distance between two individuals. As already mentioned, de Marti and Zenou (2017) model is a variation of the connection model where the cost of a link is endogenous and depends on the neighborhood structure of the two individuals involved in the link.

We go further the related literature by considering the impact of a mixed population along two dimensions (community membership and degree of farsightedness) on the stability of friendship networks. That is, we analyze how the presence of farsighted individuals can affect the long-run integration outcome and under which circumstances this can lead either to a segregated society or to a society where one community is fully or partially assimilated into the other one. By doing so, we are the first to provide a theoretical network formation model that stabilizes in the long-run the efficient network structure where the smaller community ends up fully assimilated into the larger community. ${ }^{4}$

Another strand of the literature studies the role of social networks in the assimilation of immigrants, a hot debate in the United States and in Europe. There is strong evidence showing that family, peers and communities affect assimilation decisions (see e.g. Bisin, Patacchini, Verdier and Zenou, 2016). In particular, there may be a conflict between an individual's assimilation choice and that of her peers and between an individual's assimilation choice and that of her family and community. Verdier and Zenou (2017) study the role of the immigrant network in the assimilation process of ethnic minorities. They show that, in an exogenous network, the more central minority individuals are located in the social network, the more they assimilate to the majority culture. By endogenizing the network structure, they show when the ethnic minority will integrate or not into the majority group.

The paper is organized as follows. In Section 2 we present de Marti and Zenou (2017) model of friendship networks with two communities and we look at which networks are likely to arise when all individuals are myopic. In Section 3 we introduce the concept of myopic-farsighted stable sets. In Section 4 we provide a characterization of the myopicfarsighted stable sets when intra-community costs are low. In Section 5 we consider the case where intra-community costs are intermediate. Finally, in Section 6 we conclude.

\section{Friendship networks with two communities}

We consider de Marti and Zenou (2017) model of friendship networks where individuals belong to two different communities. Individuals benefit from direct and indirect connec-

\footnotetext{
${ }^{4}$ Using data from the German Socio-Economic panel for the period 1996 to 2011, Facchini, Patacchini and Steinhardt (2015) find that first generation migrants who have a German friend are more similar to German natives than migrants who do not. In addition, the educational achievement is positively related to the likelihood of forming friendships with majority group members. Similarly, from data of the European Community Household Panel (1994-2001), de Palo, Faini and Venturini (2007) find that more educated migrants tend to socialize more intensively with the majority community.
} 
tions to others, which can be interpreted as positive externalities. These benefits decay with distance between individuals and the cost of forming links may depend on community memberships. The novelty is that individuals can now be either farsighted or myopic when deciding about the friendship links they want to form. In de Marti and Zenou (2017) all individuals were supposed to be myopic.

The set of individuals is denoted by $N=N^{M} \cup N^{F}$, where $N^{M}$ is the set of myopic individuals and $N^{F}$ is the set of farsighted individuals. Let $n$ be the total number of individuals and $n^{M} \geq 0\left(n^{F}=n-n^{M} \geq 0\right)$ be the number of myopic (farsighted) individuals. Moreover, the population is divided into two communities $N=N^{B} \cup N^{G}$, where $N^{B}$ is the blue community and $N^{G}$ is the green community. Each individual belongs to one of the two communities and the type of individual $i$ is denoted as $\tau(i) \in\left\{N^{B}, N^{G}\right\}$. We have $n=n^{B}+n^{G}$, where $n^{B}$ and $n^{G}$ denote, respectively, the number of $N^{B}$ individuals and the number of $N^{G}$ individuals in the population. Without loss of generality, the green community is the largest one: $n^{B} \leq n^{G}$.

A friendship network $g$ is a list of which pairs of individuals are linked to each other and $i j \in g$ indicates that $i$ and $j$ are linked under $g$. The complete network on the set of individuals $S \subseteq N$ is denoted by $g^{S}$ and is equal to the set of all subsets of $S$ of size 2 . It follows in particular that the empty network is denoted by $g^{\emptyset}$. The set of all possible networks on $N$ is denoted by $\mathcal{G}$ and consists of all subsets of $g^{N}$. The network obtained by adding link $i j$ to an existing network $g$ is denoted $g+i j$ and the network that results from deleting link $i j$ from an existing network $g$ is denoted $g-i j$. Let $N(g)=\{i$ |there is $j$ such that $i j \in g$ \} be the set of individuals who have at least one link in the network g. Let $N_{i}(g)=\{j \in N \mid i j \in g\}$ be the set of neighbors (or friends) of individual $i$ in $g .{ }^{5}$ Let $n_{i}(g)=\#\left(N_{i}(g)\right)$ be the number of neighbors (or friends) of individual $i$ in $g$. A path in a network $g$ between $i$ and $j$ is a sequence of individuals $i_{1}, \ldots, i_{K}$ such that $i_{k} i_{k+1} \in g$ for each $k \in\{1, \ldots, K-1\}$ with $i_{1}=i$ and $i_{K}=j$. A network $g$ is connected if for all $i \in N$ and $j \in N \backslash\{i\}$, there exists a path in $g$ connecting $i$ and $j$. A nonempty subnetwork $h \subseteq g$ is a component of $g$, if for all $i \in N(h)$ and $j \in N(h) \backslash\{i\}$, there exists a path in $h$ connecting $i$ and $j$, and for any $i \in N(h)$ and $j \in N(g), i j \in g$ implies $i j \in h$. A star network is a network such that there exists some individual $i$ (the center) who is linked to every other individual $j \neq i$ (the peripherals) and that contains no other links (i.e. $g$ is such that $N_{i}(g)=N \backslash\{i\}$ and $N_{j}(g)=\{i\}$ for all $\left.j \in N \backslash\{i\}\right)$.

A network utility function (or payoff function) is a mapping $U_{i}: \mathcal{G} \rightarrow \mathbb{R}$ that assigns to each network $g$ a utility $U_{i}(g)$ for each individual $i \in N$. A network $g \in \mathcal{G}$ is strongly efficient if $\sum_{i \in N} U_{i}(g) \geq \sum_{i \in N} U_{i}\left(g^{\prime}\right)$ for all $g^{\prime} \in \mathcal{G}$. Preferences are given by

$$
U_{i}(g)=\sum_{j \neq i} \delta^{t(i, j)}-\sum_{j \in N_{i}(g)} c_{i j}(g)
$$

where $t(i j)$ is the number of links in the shortest path between $i$ and $j$ (setting $t(i j)=\infty$

\footnotetext{
${ }^{5}$ Throughout the paper we use the notation $\subseteq$ for weak inclusion and $\varsubsetneqq$ for strict inclusion. Finally, \# will refer to the notion of cardinality.
} 
if there is no path between $i$ and $j$ ), $0<\delta<1$ is the benefit from a connection that decreases with the distance of the relationship, ${ }^{6}$ and $c_{i j}(g)>0$ is the cost for individual $i$ of maintaining a direct link with $j$. The cost of forming one link may vary as a function of the type of individuals connected by such link.

Definition 1 (de Marti and Zenou, 2017). Given a network $g$, the rate of exposure of individual $i$ to their own community $\tau(i)$ is

$$
e_{i}^{\tau(i)}(g)= \begin{cases}n_{i}^{\tau(i)}(g) /\left(n_{i}(g)-1\right) & \text { if } 0<n_{i}^{\tau(i)}(g)<n_{i}(g) \\ 0 & \text { if } n_{i}^{\tau(i)}(g)=0\end{cases}
$$

where $n_{i}^{\tau(i)}(g)$ is the number of $i$ 's same-type friends in network $g$ while $n_{i}(g)$ is the total number of $i$ 's friends in network $g$.

Let $c$ and $C$ be strictly positive parameters, $c>0$ and $C>0$. The cost for individual $i$ of maintaining a link with $j, c_{i j}(g)$, depends on whether $i$ and $j$ belong or not to the same community:

$$
c_{i j}(g)=\left\{\begin{array}{ll}
c & \text { if } \tau(i)=\tau(j) \\
c+e_{i}^{\tau(i)}(g) \cdot e_{j}^{\tau(j)}(g) \cdot C & \text { if } \tau(i) \neq \tau(j)
\end{array} .\right.
$$

Such cost function assumes that it is less costly to interact with someone of the same type (intra-community cost) than with someone of a different type (inter-community cost). Notice that $C$ is not present in the cost of a link between individuals of the same community. But, $C$ becomes an additional cost when two individuals from different communities, having links with individuals of their own community, form a link between them. For instance, if a green individual has only green friends, then it will be more costly for her to interact with a blue individual that has mostly blue friends. However, the more similar the friendship composition of two individuals of different types, the easier it is for them to interact. If at least $i$ or $j$ has no friends of the same type (i.e., $e_{i}^{\tau(i)}=0$ or $\left.e_{j}^{\tau(j)}=0\right)$, then it is equally costly for them to interact with someone of the opposite type as with someone of the same type (i.e., the cost is $c$ in both cases). ${ }^{7}$ In Figure 1 we depict a friendship network among seven individuals and two communities $\left(N^{G}=\{1,2,3,4\}\right.$, $\left.N^{B}=\{5,6,7\}\right)$ with a bridge link between both communities. Green individuals are represented by solid circles while blue individuals are represented by circles. For instance, green individual 4's payoff is equal to $4 \delta+2 \delta^{2}-4 c-C$ since $e_{4}^{\tau(4)}=3 /(4-1)=1$ and $e_{7}^{\tau(7)}=2 /(3-1)=1$.

We now describe some prominent network configurations in the case of friendship networks with communities. Let $g_{\text {assi,green }}$ denote the network where all members of the

\footnotetext{
${ }^{6}$ It is similar to the connections model introduced by Jackson and Wolinsky (1996).

${ }^{7}$ In the definition of the rate of exposure (see the expression (1)), we subtract 1 in the denominator because, when computing the cost of a given bridge link between communities, this bridge link is not included in the computation of the cost. What is relevant for the cost is the rate of exposure according to the rest of the connections of each of the two individuals involved in the bridge link.
} 


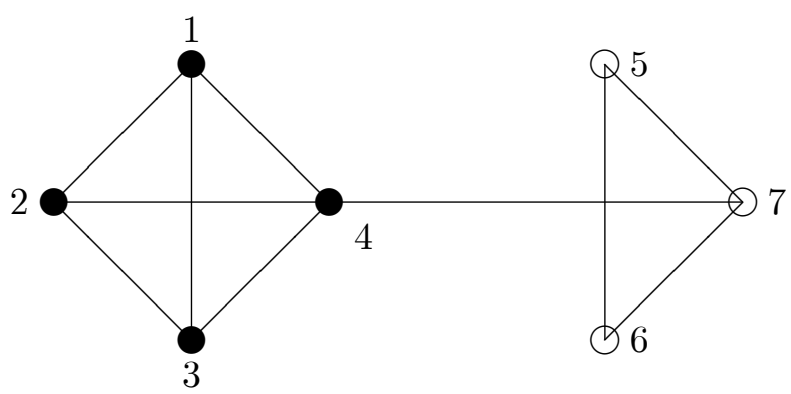

Figure 1: A bridge link between both communities. Greens are represented by solid circles while blues are represented by circles.

blue community are fully assimilated to the dominant (or larger) green community. That is, each green individual is linked to all other (green and blue) individuals while each blue individual is only linked to all green individuals. Formally, $g_{\text {assi,green }}=g^{N^{G}} \cup\{i j \mid i \in$ $\left.N^{G}, j \in N^{B}\right\}$. In Figure 2 we depict $g_{\text {assi,green }}$ for $N^{G}=\{1,2,3,4\}$ and $N^{B}=\{5,6\}$. Similarly, let $g_{\text {assi,blue }}$ denote the network where all members of the green community are fully assimilated to the smaller blue community. That is, each blue individual is linked to all other (green and blue) individuals while each green individual is only linked to all blue individuals. Formally, $g_{\text {assi,blue }}=g^{N^{B}} \cup\left\{i j \mid i \in N^{B}, j \in N^{G}\right\}$. In Figure 3 we depict $g_{\text {assi,blue }}$ for $N^{G}=\{1,2,3,4\}$ and $N^{B}=\{5,6\}$. Let $g_{\text {int }}$ denote the complete integration network where both communities are fully intra-connected and fully interconnected: $g_{\text {int }}=g^{N}$ and is depicted in Figure 4. Let $g_{\text {seg }}$ denote the complete segregation network where both communities are fully intra-connected but isolated of each other: $g_{\text {seg }}=g^{N^{G}} \cup g^{N^{B}}$ and is depicted in Figure 5 .

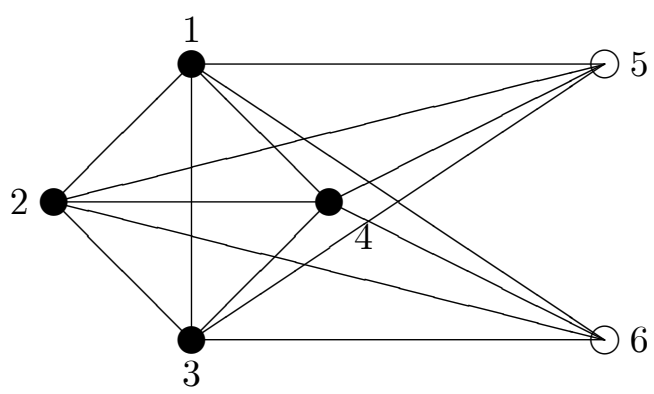

Figure 2: The blue community is fully assimilated within the green community.

de Marti and Zenou (2017) adopt the notion of pairwise stability, introduced by Jackson and Wolinsky (1996), to study the networks that will be formed at equilibrium. A 


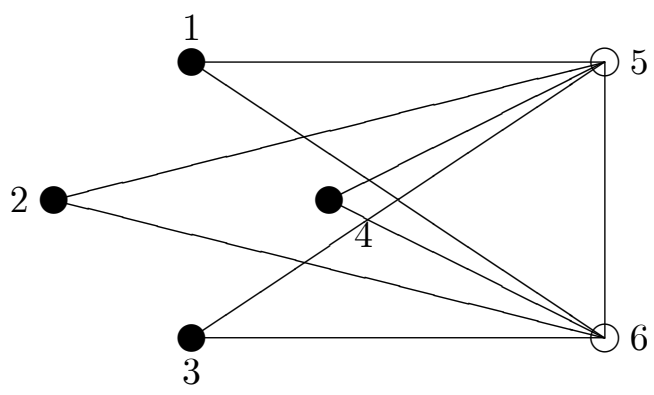

Figure 3: The green community is fully assimilated within the blue community.

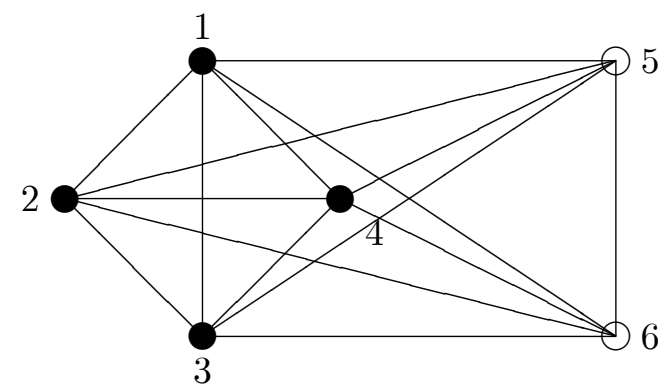

Figure 4: Both communities are fully integrated.
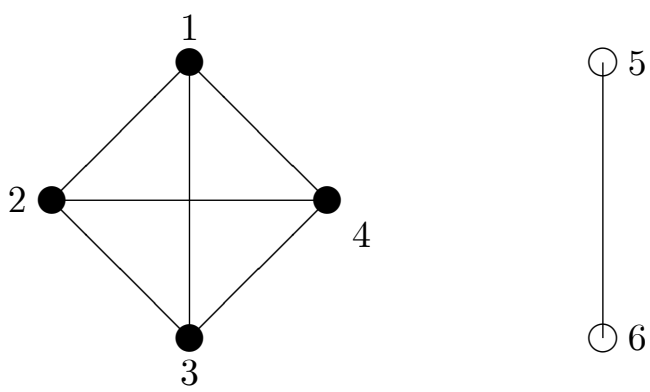

Figure 5: Both communities are segregated. 
network is pairwise stable if no individual benefits from deleting a link and no two individuals benefit from adding a link between them. Formally, a network $g \in \mathcal{G}$ is pairwise stable if (i) for all $i j \in g, U_{i}(g) \geq U_{i}(g-i j)$ and $U_{j}(g) \geq U_{j}(g-i j)$, (ii) for all $i j \notin g$, if $U_{i}(g)<U_{i}(g+i j)$ then $U_{j}(g)>U_{j}(g+i j)$. Let $P$ be the set of pairwise stable networks. Pairwise stability presumes that individuals are myopic: they do not anticipate that other individuals may react to their changes. Denote $\Delta \equiv \delta-\delta^{2}-c$. De Marti and Zenou (2017) find necessary and sufficient conditions for the stability of the complete integration (segregation) network.

Proposition 1 (de Marti and Zenou, 2017). Assume low intra-community costs, $0<\Delta$ or $c<\delta-\delta^{2}$.

(i) The complete integration network $g_{\text {int }}=g^{N}$ is pairwise stable if and only if

$$
C<\frac{(n-2)^{2}(n-3)}{n^{G}\left(n^{G}-1\right)^{2}} \Delta
$$

(ii) The complete segregation network $g_{\text {seg }}=g^{N^{G}} \cup g^{N^{B}}$ is pairwise stable 8 if and only if

$$
C>\Delta+n^{B} \cdot \delta^{2}
$$

We now show that friendship networks where one community is fully or partially assimilated to the other community can also emerge in the long run when intra-community costs are low. In Figure 6 (7) we depict a network where one blue (green) individual is assimilated to the green (blue) community, while the rest of blue (green) individuals are isolated. All the proofs not in the main text can be found in the appendix.

Proposition 2. Assume low intra-community costs, $0<\Delta$ or $c<\delta-\delta^{2}$.

(i) The network $g_{\text {assi,green }}=g^{N^{G}} \cup\left\{i j \mid i \in N^{G}, j \in N^{B}\right\}$ where all the blue community is fully assimilated to the green community is pairwise stable if and only if

$$
C>\frac{(n-2)}{\left(n^{G}-1\right)} \Delta
$$

(ii) The network $g_{\text {assi,blue }}=g^{N^{B}} \cup\left\{i j \mid i \in N^{B}, j \in N^{G}\right\}$ where all the green community is fully assimilated to the blue community is pairwise stable if and only if

$$
C>\frac{(n-2)}{\left(n^{B}-1\right)} \Delta
$$

\footnotetext{
${ }^{8}$ Notice that there is a typo in de Marti and Zenou's original condition: $C>\Delta+n^{G} \delta^{2}$ has to be replaced by $C>\Delta+n^{B} \delta^{2}$.
} 
(iii) Take any $N^{B_{1}} \varsubsetneqq N^{B}$ such that $1 \leq n^{B_{1}} \leq n^{B}-2$. The network $g_{\text {passi, green }}=$ $g^{N^{G}} \cup g^{N^{B} \backslash N^{B_{1}}} \cup\left\{i j \mid i \in N^{G}, j \in N^{B_{1}}\right\}$ where $n^{B_{1}}$ blue individuals are assimilated to the green individuals and all other blue individuals are intra-connected and segregated is pairwise stable if and only if

$$
C>\frac{\left(n^{G}+n^{B_{1}}-1\right)}{\left(n^{G}-1\right)}\left(\Delta+\left(n^{B}-n^{B_{1}}\right) \delta^{2}\right) ;
$$

(iv) Take any $N^{G_{1}} \varsubsetneqq N^{G}$ such that $1 \leq n^{G_{1}} \leq n^{G}-2$. The network $g_{\text {passi,blue }}=g^{N^{B}} \cup$ $g^{N^{G} \backslash N^{G_{1}}} \cup\left\{i j \mid i \in N^{B}, j \in N^{G_{1}}\right\}$ where $n^{G_{1}}$ green individuals are assimilated to the blue individuals and all other green individuals are intra-connected and segregated is pairwise stable if and only if

$$
C> \begin{cases}\widehat{C}_{1} & \text { if } n^{G_{1}} \leq \frac{1}{2}\left(n^{G}-n^{B}\right) ; \\ \widehat{C}_{2} & \text { if } n^{G_{1}}>\frac{1}{2}\left(n^{G}-n^{B}\right) ;\end{cases}
$$

where

$$
\begin{aligned}
& \widehat{C}_{1}=\max \left\{\frac{\left(n^{B}+n^{G_{1}}-1\right)}{\left(n^{B}-1\right)}\left(\Delta+\left(n^{B}+n^{G_{1}}\right) \delta^{2}\right), \frac{\left(n^{B}+n^{G_{1}}-2\right)}{\left(n^{B}-1\right)}\left(\Delta+\left(n^{G}-n^{G_{1}}\right) \delta^{2}\right)\right\} \\
& \widehat{C}_{2}=\frac{\left(n^{B}+n^{G_{1}}-1\right)}{\left(n^{B}-1\right)}\left(\Delta+\left(n^{G}-n^{G_{1}}\right) \delta^{2}\right) .
\end{aligned}
$$
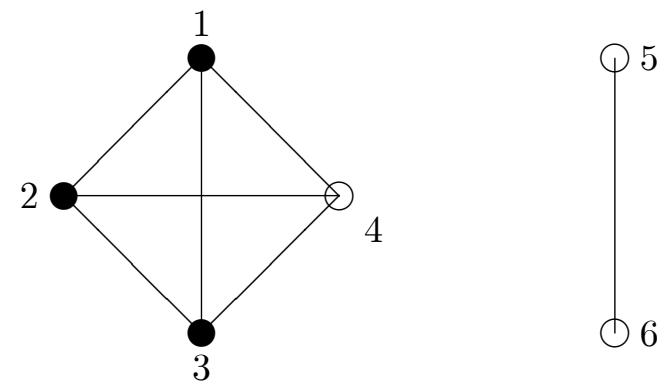

Figure 6: One blue individual is assimilated to the green community while the rest of blue individuals are segregated.

Proposition 2 of de Marti and Zenou (2017) points out that if intra-community costs are low, some asymmetric network configurations can also be pairwise stable: (i) the network in which both communities are fully intra-connected and where there is only one bridge link (see Figure 1), (ii) the network in which both communities are fully intra-connected, where each blue individual has one and only one bridge link, and where each green individual has at most one bridge link, and (iii) the network in which both communities are fully intra-connected and with a unique blue individual connected with 

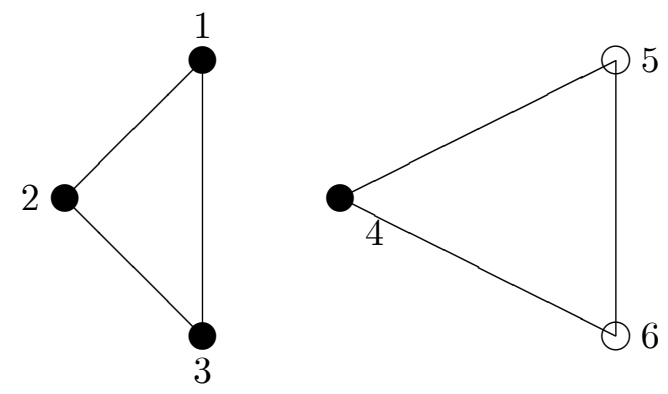

Figure 7: One green individual is assimilated to the blue community while the other green individuals are segregated.

all green individuals. In the appendix we show that even more friendship networks can be pairwise stable. For instance, the network in which both communities are fully intraconnected and in which one green individual is linked to all blue individuals.

In terms of strong efficiency considerations, one might wonder which of the pairwise stable networks is better from a social point of view. de Marti and Zenou (2017) only compare the efficiency of the complete integrated network and the complete segregated network, and they conclude that, depending on the size of relative communities, one cannot plead for integrated or segregated socialization patterns a priori. We next compare in terms of strong efficiency the complete integrated network, the complete segregated network, and the networks with full or partial assimilation.

Proposition 3. Assume low intra-community costs, $0<\Delta$ or $c<\delta-\delta^{2}$. Let

$$
C^{*}=\frac{(n-2)^{2}}{2 n^{G}\left(n^{G}-1\right)} \Delta .
$$

(i) If $C<C^{*}$, the complete integrated network $g_{\text {int }}$ is strongly efficient.

(ii) If $C>C^{*}$, the network $g_{\text {assi,green }}$ in which all blue individuals are fully assimilated into the dominant green community and all green individuals are fully inter- and intra-connected is strongly efficient.

(iii) The complete segregated network $g_{\text {seg }}$ is never strongly efficient for any value of $C$.

Thus, contrary to de Marti and Zenou (2017), we obtain that the complete segregated network is never strongly efficient. Only the complete integrated network and the network in which the blue individuals are fully assimilated into the dominant green community are strongly efficient. Indeed, the efficiency of one or the other network depends on $C$, which affects the exposure effect that the formation of a new link has on the exposure rates of the individuals involved in it. The formation of a link between two individuals from 
different communities (the same community), has a positive (negative) exposure effect for the individuals involved in it because the decrease (increase) in the rate of exposure of each of these individuals to their own community will reduce (increase) their intercommunity costs that are proportional to $C$. When $C$ is small enough (close to 0 ), the difference between the inter-community and the intra-community costs is negligible and then one can consider that the entire population belong to only one community. When this is the case, Proposition 2 in Jackson and Wolinsky (1996) is applicable and the complete integrated network is both pairwise stable and strongly efficient. When $C$ increases, the inter-community costs might overcome the benefits derived from connecting to the other community. When this is the case, it becomes preferable to avoid the inter-community costs, making efficient the network in which the blue individuals (without any link to other blue individuals) are fully assimilated into the dominant green community.

Proposition 3 and Proposition 5 in de Marti and Zenou (2017) provide conditions for the stability of some type of networks when intra-community costs are intermediate (i.e. $\delta-\delta^{2}<c<\delta-\delta^{3}$ or $\delta-\delta^{2}<c<\delta$ ): (i) the bipartite network in which all green individuals are linked to all blue individuals, and in which all blue individuals are linked to all green individual, (ii) the network with two disconnected star-shaped communities, (iii) the network where the star-shaped communities are connected through their central individuals, (iv) the network where the star-shaped communities are connected through their peripheral individuals, and (v) the network where the star-shaped communities are connected through their central individuals and through their peripheral individuals. However, all those networks are not efficient. In fact, a star network encompassing all individuals is pairwise stable and is strongly efficient.

Proposition 4. Assume intermediate intra-community costs, $\delta-\delta^{2}<c<\delta$. A star network is both pairwise stable and strongly efficient.

Up to now it has been assumed that all individuals were myopic in the friendship network formation. We next allow the population to include not only myopic individuals but also farsighted ones. Farsighted individuals are able to anticipate that once they add or delete some links, other individuals could add or delete links afterwards.

\section{$3 \quad$ Myopic-farsighted stable sets}

We adopt the notion of myopic-farsighted stable set introduced by Herings, Mauleon and Vannetelbosch (2020) to determine the networks that are stable when some individuals are myopic while others are farsighted. ${ }^{9}$ A set of networks $G$ is said to be a myopic-farsighted

\footnotetext{
${ }^{9}$ See Chwe (1994), Herings, Mauleon and Vannetelbosch (2009), Mauleon, Vannetelbosch and Vergote (2011), Ray and Vohra (2015, 2019), Roketskiy (2018) for definitions of the farsighted stable set when individuals are farsighted. Alternative notions of farsightedness for network formation are suggested by Dutta, Ghosal and Ray (2005), Dutta and Vohra (2017), Herings, Mauleon and Vannetelbosch (2019), Page, Wooders and Kamat (2005), Page and Wooders (2009) among others.
} 
stable set if it satisfies the following two types of stability. Internal stability: No network in $G$ is dominated by any other network in $G$. External stability: Every network not in $G$ is dominated by some network in $G$. A network $g^{\prime}$ is said to be dominated by a network $g$ if there is a myopic-farsighted improving path from $g^{\prime}$ to $g$.

A myopic-farsighted improving path is a sequence of distinct networks that can emerge when farsighted individuals form or delete links based on the improvement the end network offers relative to the current network while myopic individuals form or delete links based on the improvement the resulting network offers relative to the current network. Since we only allow for pairwise deviations, each network in the sequence differs from the previous one in that either a new link is formed between two individuals or an existing link is deleted. If a link is deleted, then it must be that either a myopic individual prefers the resulting network to the current network or a farsighted individual prefers the end network to the current network. If a link is added between some myopic individual $i$ and some farsighted individual $j$, then the myopic individual $i$ must prefer the resulting network to the current network and the farsighted individual $j$ must prefer the end network to the current network. ${ }^{10}$

Definition 2. A myopic-farsighted improving path from a network $g$ to a network $g^{\prime}$ is a finite sequence of distinct networks $g_{1}, \ldots, g_{K}$ with $g_{1}=g$ and $g_{K}=g^{\prime}$ such that for any $k \in\{1, \ldots, K-1\}$ either

(i) $g_{k+1}=g_{k}-i j$ for some $i j$ such that $U_{i}\left(g_{k+1}\right)>U_{i}\left(g_{k}\right)$ and $i \in N^{M}$ or $U_{j}\left(g_{K}\right)>U_{j}\left(g_{k}\right)$ and $j \in N^{F}$; or

(ii) $g_{k+1}=g_{k}+i j$ for some $i j$ such that $U_{i}\left(g_{k+1}\right)>U_{i}\left(g_{k}\right)$ and $U_{j}\left(g_{k+1}\right) \geq U_{j}\left(g_{k}\right)$ if $i, j \in$ $N^{M}$, or $U_{i}\left(g_{K}\right)>U_{i}\left(g_{k}\right)$ and $U_{j}\left(g_{K}\right) \geq U_{j}\left(g_{k}\right)$ if $i, j \in N^{F}$, or $U_{i}\left(g_{k+1}\right) \geq U_{i}\left(g_{k}\right)$ and $U_{j}\left(g_{K}\right) \geq U_{j}\left(g_{k}\right)$ (with one inequality holding strictly) if $i \in N^{M}, j \in N^{F}$.

If there exists a myopic-farsighted improving path from a network $g$ to a network $g^{\prime}$, then we write $g \rightarrow g^{\prime}$. The set of all networks that can be reached from a network $g \in \mathcal{G}$ by a myopic-farsighted improving path is denoted by $\phi(g), \phi(g)=\left\{g^{\prime} \in \mathcal{G} \mid g \rightarrow g^{\prime}\right\}$. When all individuals are myopic, our notion of myopic-farsighted improving path reverts to Jackson and Watts (2002) notion of improving path. When all individuals are farsighted, our notion of myopic-farsighted improving path reverts to Jackson (2008) and Herings, Mauleon and Vannetelbosch (2009) notion of farsighted improving path. A set of networks $G$ is a myopic-farsighted stable set if the following two conditions hold. Internal stability: for any two networks $g$ and $g^{\prime}$ in the myopic-farsighted stable set $G$ there is no myopic-farsighted improving path from $g$ to $g^{\prime}$ (and vice versa). External stability: for

\footnotetext{
${ }^{10}$ Along a myopic-farsighted improving path, myopic players do not care whether other players are myopic or farsighted. They behave as if all players are myopic and they compare their resulting network's payoff to their current network's payoff for taking a decision. However, farsighted players know exactly who is farsighted and who is myopic and they compare their end network's payoff to their current network's payoff for taking a decision.
} 
every network $g$ outside the myopic-farsighted stable set $G$ there is a myopic-farsighted improving path leading to some network $g^{\prime}$ in the myopic-farsighted stable set $G$ (i.e. there is $g^{\prime} \in G$ such that $\left.g \rightarrow g^{\prime}\right)$.

Definition 3. A set of networks $G \subseteq \mathcal{G}$ is a myopic-farsighted stable set if: (IS) for every $g, g^{\prime} \in G$, it holds that $g^{\prime} \notin \phi(g)$; and (ES) for every $g \in \mathcal{G} \backslash G$, it holds that $\phi(g) \cap G \neq \emptyset$.

When all individuals are farsighted, the myopic-farsighted stable set is simply the farsighted stable set as defined in Herings, Mauleon and Vannetelbosch (2009) or Ray and Vohra (2015). When all individuals are myopic, the myopic-farsighted stable set boils down to the pairwise CP vNM set as defined in Herings, Mauleon, and Vannetelbosch (2017) for two-sided matching problems. ${ }^{11}$

When all individuals are myopic, Jackson and Watts (2002) define the notions of cycle and closed cycle. A set of networks $\mathcal{C}$, form a cycle if for any $g \in \mathcal{C}$ and $g^{\prime} \in \mathcal{C}$ there exists an improving path connecting $g$ to $g^{\prime}$. A cycle $\mathcal{C}$ is a closed cycle if no network in $\mathcal{C}$ lies on an improving path leading to a network that is not in $\mathcal{C}$. Luo, Mauleon and Vannetelbosch (2020) characterize the myopic-farsighted stable set when all individuals are myopic (i.e. $N=N^{M}$ ): a set of networks is a myopic-farsighted stable set if and only if it consists of all pairwise stable networks and one network from each closed cycle.

Similar to pairwise stability, one may alternatively look for networks that are immune to deviations by myopic and farsighted individuals. A network $g \in \mathcal{G}$ is myopicfarsightedly pairwise stable if $\phi(g)=\emptyset$. The set of myopic-farsightedly pairwise stable networks is denoted by $P_{M F}$. When $N=N^{F}$ it reverts to Jackson (2008) set of farsightedly pairwise stable networks. Since $P_{M F} \subseteq P$, it is not surprising that the set $P_{M F}$ is often empty.

\section{Low intra-community costs}

Suppose now that the population of individuals is mixed in terms of their degree of farsightedness. We first show that if the intra- and inter-community costs are low, i.e. $c+n^{G} C<\delta-\delta^{2}$, then the complete integrated network is stable whatever the composition of the population in terms of farsightedness.

Proposition 5. Assume low intra-community costs and low inter-community costs, $n^{G} C<$ $\Delta$ or $c+n^{G} C<\delta-\delta^{2}$. The set $G=\left\{g_{\text {int }}\right\}$, where $g_{\text {int }}=g^{N}$, is a myopic-farsighted stable set.

Proof. The set $G=\left\{g_{\text {int }}\right\}$, where $g_{\text {int }}=g^{N}$, satisfies (IS) in Definition 3 since it is a singleton set. We now show that it also satisfies (ES).

ES. Take any network $g \neq g_{\text {int }}$. Since $n^{G} C<\Delta$ or $c+n^{G} C<\delta-\delta^{2}$, it follows that

\footnotetext{
${ }^{11}$ The pairwise CP vNM set follows the approach by Page and Wooders (2009) who define the stable set with respect to path dominance, i.e. the transitive closure of $\phi$.
} 
$U_{i}(g+i j)>U_{i}(g)$ and $U_{j}(g+i j)>U_{j}(g)$ as well as $U_{i}\left(g^{N}\right) \geq U_{i}(g+i j)>U_{i}(g)$ and $U_{j}\left(g^{N}\right) \geq U_{j}(g+i j)>U_{j}(g)$. Hence, the sequence starting at $g_{1}=g$, followed by $g_{k+1}=g_{k}+i j$ with $i j \in g^{N} \backslash g^{k}$, for $k=1,2 \ldots$, and ending at $g_{K}=g^{N}$, is a sequence along which $U_{i}\left(g_{k}+i j\right)>U_{i}\left(g_{k}\right), U_{j}\left(g_{k}+i j\right)>U_{j}\left(g_{k}\right), U_{i}\left(g^{N}\right)>U_{i}\left(g_{k}\right)$ and $U_{j}\left(g^{N}\right)>U_{j}\left(g_{k}\right)$. Thus, this sequence is a myopic-farsighted improving path from $g$ to $g^{N}$ whatever the composition of the population in terms of myopia and farsightedness (i.e. $N^{M}$ and $N^{F}$ ), and $G=\left\{g_{\text {int }}\right\}$ satisfies (ES).

When all individuals are myopic each myopic-farsighted stable set contains all pairwise networks. Hence, many inefficient friendship networks can emerge in the long run when both communities are composed of only myopic individuals.

We next focus on three particular cases: (1) all individuals in the larger green community are farsighted, while all individuals in the smaller blue community are myopic; (2) all individuals in the larger green community are myopic, while all individuals in the smaller blue community are farsighted; (3) all individuals in both communities are farsighted.

\subsection{Greens are farsighted, blues are myopic}

We now show that if the dominant group (green community) is farsighted while the other group (blue community) is myopic, the friendship network where the blue individuals end up assimilated to the dominant green community is the unique stable network and is strongly efficient. Let $\bar{C}_{1}$ be the upper bound on the inter-community cost parameter $C$ such that a blue individual has no incentive to cut a link with another blue individual in the complete integrated network, and it is given by

$$
\bar{C}_{1}=\frac{(n-2)^{2}(n-3)}{n^{G}\left(n^{G}-1\right)^{2}} \Delta .
$$

Thus, if $C>\bar{C}_{1}$, each myopic blue individual has an incentive to delete some link to another blue individual in the complete integrated network $g^{N}$.

Proposition 6. Assume low intra-community costs, $0<\Delta$ or $c<\delta-\delta^{2}$ and large intercommunity costs, $C>\bar{C}_{1}$. Assume all individuals in the blue community are myopic, $N^{M}=N^{B}$, and all individuals in the green community are farsighted, $N^{F}=N^{G}$. Then, the set $G=\left\{g_{\text {assi,green }}\right\}$, where $g_{\text {assi,green }}=g^{N^{G}} \cup\left\{i j \mid i \in N^{G}, j \in N^{B}\right\}$, is the unique myopic-farsighted stable set.

Proof. The set $G=\left\{g_{\text {assi,green }}\right\}$ satisfies (IS) in Definition 3 since it is a singleton set. We now show that it also satisfies (ES).

ES. Take any network $g \neq g_{\text {assi,green }}$. We build in steps a myopic-farsighted improving path from $g$ to $g_{\text {assi,green }}$.

Step 0: If $g$ is such that blue individuals have links among themselves, i.e., $g \cap g^{N^{B}} \neq \emptyset$ then go to Step 1. Otherwise, starting from $g$, green individuals first build all the missing links between green individuals to reach $g^{\prime}=g \cup g^{N^{G}}$ looking forward to $g_{\text {assi,green }}$, where 
they obtain their highest possible payoff given $c<\delta-\delta^{2}, U_{i}\left(g_{\text {assi,green }}\right)=(n-1)(\delta-c)$. From $g^{\prime}$ green individuals build all the missing links with blue individuals to finally reach $g^{\prime \prime}=g^{\prime} \cup\left\{i j \mid i \in N^{G}, j \in N^{B}\right\}=g_{\text {assi,green }}$. Since $c<\delta-\delta^{2}$ and $g^{\prime \prime} \cap g^{N^{B}}=\emptyset$, blue individuals are assimilated to the green community in $g^{\prime \prime}$ and they are not affected by $C$ and so they have incentives to add the links with the green individuals.

Step 1: Starting in $g$, green individuals who are all farsighted $\left(N^{F}=N^{G}\right)$ delete successively all the links (if any) they have with green individuals looking forward to $g_{\text {assi,green }}$, where they obtain their highest possible payoff given $c<\delta-\delta^{2}, U_{i}\left(g_{\text {assi,green }}\right)=$ $(n-1)(\delta-c)$. We reach the network $g^{\prime}=g \backslash g^{N^{G}}$ where there are no links between green individuals.

Step 2: From $g^{\prime}=g \backslash g^{N^{G}}$, since $c<\delta-\delta^{2}$, blue individuals who are all myopic have incentives to build all the links with the green individuals. Green individuals who are looking forward $g_{\text {assi,green }}$ prefer the end network to the current one. We reach the network $g^{\prime \prime}=g^{\prime} \cup\left\{i j \mid i \in N^{G}, j \in N^{B}\right\}$ where all possible links between blue and green individuals are formed.

Step 3: From $g^{\prime \prime}=g^{\prime} \cup\left\{i j \mid i \in N^{G}, j \in N^{B}\right\}$, since $c<\delta-\delta^{2}$, blue individuals who are all myopic have incentives to build all the missing links between the blue individuals. We reach the network $g^{\prime \prime \prime}=g^{\prime \prime} \cup g^{N^{B}}$ where all the green individuals are assimilated to the blue community and the blue community is fully intra-connected. In fact, $g^{\prime \prime \prime}=g_{\text {assi,blue }}$ and all green individuals prefer $g_{\text {assi,green }}$ to $g_{\text {assi,blue }}$.

Step 4: From $g^{\prime \prime \prime}=g^{\prime \prime} \cup g^{N^{B}}$, green individuals who are all farsighted and look forward towards $g_{\text {assi,green }}$ build all the links between the green individuals to reach $g^{N}$.

Step 5: From the complete network $g^{N}$, since $C>\bar{C}_{1}$, blue individuals who are myopic have incentives to delete successively all the links they have with other blue individuals to finally reach the network $g_{\text {assi,green }}=g^{N} \backslash g^{N^{B}}$. The condition $C>\bar{C}_{1}$ guarantees that, along the myopic-farsighted improving path starting at $g_{1}=g^{N}$, followed by $g_{k+1}=g_{k}-i j$ with $i j \in g_{k}$ and $i, j \in N^{B}$ for $k \geq 1$, and ending at $g_{K}=g^{N} \backslash g^{N^{B}}=g_{\text {assi,green }}$, all the blue individuals have myopic incentives to delete their links with other blue individuals. Indeed, consider a sequence starting at $g_{1}=g^{N}$, followed by $g_{k+1}=g_{k}-i j$ with $i \in N^{B}, j \in N_{i}\left(g_{k}\right) \cap N^{B}$, for $k=1, \ldots n^{B}-1$. Along this sequence, a blue individual $i$ successively deletes all her links with the other blue individuals and she has incentives to cut her $k$ th link to some blue individual if and only if

$$
C>\Delta \frac{(n-2)(n-1-k)(n-2-k)}{n^{G}\left(n^{G}-1\right)^{2}} .
$$

This condition is satisfied since $C>\bar{C}_{1}$ and

$$
\bar{C}_{1}=\Delta \frac{(n-2)^{2}(n-3)}{n^{G}\left(n^{G}-1\right)^{2}} \geq \Delta \frac{(n-2)(n-1-k)(n-2-k)}{n^{G}\left(n^{G}-1\right)^{2}} .
$$

Farsighted green individuals obtain their highest possible payoff in $g_{\text {assi,green }}$ and myopic blue individuals have no incentive to delete any link nor to add a new link since $C>\bar{C}_{1}$ and $c<\delta-\delta^{2}$. Hence, $\phi\left(g_{\text {assi,green }}\right)=\emptyset$. So, since $\phi(g) \cap\left\{g_{\text {assi,green }}\right\} \neq \emptyset$ for all $g \neq g_{\text {assi,green }}$ 
and $\phi\left(g_{\text {assi,green }}\right)=\emptyset$, the set $G=\left\{g_{\text {assi,green }}\right\}$ is the unique myopic-farsighted stable set (any other set would violate (IS) and/or (ES)).

Remark that since $\phi(g) \cap\left\{g_{\text {assi,green }}\right\} \neq \emptyset$ for all $g \neq g_{\text {assi,green }}$ and $\phi\left(g_{\text {assi,green }}\right)=\emptyset$, the network $g_{\text {assi,green }}$ is the unique myopic-farsightedly pairwise stable network, i.e., $P_{M F}=$ $\left\{g_{\text {assi,green }}\right\}$.

\subsection{Greens are myopic, blues are farsighted}

However, when the dominant green community is myopic and the blue community is farsighted, a conflict between stability and efficiency can arise. Let $\bar{C}_{2}$ be the upper bound on the inter-community cost parameter $C$ such that a green individual has no incentive to delete a link with another green individual in the complete integrated network, and it is given by

$$
\bar{C}_{2}=\frac{(n-2)^{2}(n-3)}{n^{B}\left(n^{B}-1\right)^{2}} \Delta .
$$

Thus, if $C>\bar{C}_{2}$, each myopic green individual has an incentive to delete some link to another green individual in the complete integrated network $g^{N}$.

Proposition 7. Assume low intra-community costs, $0<\Delta$ or $c<\delta-\delta^{2}$ and large intercommunity costs, $C>\bar{C}_{2}$. Assume all individuals in the blue community are farsighted, $N^{F}=N^{B}$, and all individuals in the green community are myopic, $N^{M}=N^{G}$. Then, the set $G=\left\{g_{\text {assi,blue }}\right\}$, where $g_{\text {assi,blue }}=g^{N^{B}} \cup\left\{i j \mid i \in N^{B}, j \in N^{G}\right\}$, is the unique myopic-farsighted stable set.

The proof of Proposition 7 is similar to the proof of Proposition 6 by just switching blue individuals for green ones and vice versa. For completeness, the proof of Proposition 7 can be found in the appendix. So, when $C$ is large enough $\left(C>\bar{C}_{2}\right)$, the efficient network in which the farsighted blue individuals are fully assimilated into the green community ${ }^{12}$ is not stable. Farsighted blue individuals stabilize the opposite network in which the myopic green individuals are fully assimilated into the blue community. Remark that the network $g_{\text {assi,blue }}=g^{N^{B}} \cup\left\{i j \mid i \in N^{B}, j \in N^{G}\right\}$ is the unique myopic-farsightedly pairwise stable network, i.e., $P_{M F}=\left\{g_{\text {assi,blue }}\right\}$.

\subsection{Greens and blues are farsighted}

Proposition 8. Assume low intra-community costs, $0<\Delta$ or $c<\delta-\delta^{2}$ and intercommunity costs, $C>0$. Assume all individuals are farsighted, $N^{F}=N$. Then, the set $G=\left\{g_{\text {assi,green }}\right\}$ is a myopic-farsighted stable set.

\footnotetext{
${ }^{12}$ Since $C^{*}<\bar{C}_{1}<\bar{C}_{2}$ the network in which the farsighted blue individuals are fully assimilated into the green community is the efficient network.
} 
Proof. The set $G=\left\{g_{\text {assi,green }}\right\}$ satisfies (IS) in Definition 3 since it is a singleton set. We now show that it also satisfies (ES).

ES. Take any network $g \neq g_{\text {assi,green }}$. We build in steps a myopic-farsighted improving path from $g$ to $g_{\text {assi,green }}$.

Step 0: If $g$ is such that $g \cap g^{N^{B}} \neq \emptyset$ then go to Step 1. Otherwise, starting from $g$, green individuals first build all the missing links between green individuals to reach $g^{\prime}=g \cup g^{N^{G}}$ looking forward to $g_{\text {assi,green }}$, where they obtain their highest possible payoff given $c<\delta-\delta^{2}$ and $C>0, U_{i}\left(g_{\text {assi,green }}\right)=(n-1)(\delta-c)$. From $g^{\prime}$ green individuals build all the missing links with blue individuals to finally reach $g^{\prime \prime}=g^{\prime} \cup\left\{i j \mid i \in N^{G}, j \in N^{B}\right\}=g_{\text {assi,green }}$. Since $c<\delta-\delta^{2}$ and $g^{\prime \prime} \cap g^{N^{B}}=\emptyset$, blue individuals are assimilated to the green community in $g^{\prime \prime}$ and they are not affected by $C$ and so they have incentives to add the links with the green individuals looking forward to $g_{\text {assi,green }}$.

Step 1: Starting in $g$, green individuals who are all farsighted $\left(N^{F}=N\right)$ delete successively all the links (if any) they have with green and blue individuals looking forward to $g_{\text {assi,green }}$, where they obtain their highest possible payoff given $c<\delta-\delta^{2}$ and $C>0$, $U_{i}\left(g_{\text {assi,green }}\right)=(n-1)(\delta-c)$. We reach the network $g^{\prime}=g \cap g^{N^{B}}$ where all the links involving green individuals in $g$ have been deleted. Thus, $g^{\prime} \subseteq g^{N^{B}}$.

Step 2: From $g^{\prime}=g \cap g^{N^{B}}$, since $n^{G} \geq n^{B}$, all blue individuals who are all farsighted prefer $g_{\text {assi,green }}$ to $g^{\prime}$ and so are ready to delete all their links looking forward to $g_{\text {assi,green }}$. We reach the empty network $g^{\emptyset}$.

Step 3: From the empty network $g^{\emptyset}$ green individuals and blue individuals who are farsighted and look forward to $g_{\text {assi,green }}$ build all the links in $g^{N^{G}} \cup\left\{i j \mid i \in N^{G}, j \in N^{B}\right\}$ to finally reach the network $g_{\text {assi,green }}$. Since along the myopic-farsighted improving blue individuals have no links to other blue individuals, the payoffs of both green and blue individuals are not affected by $C$. So, each time they add a link they all prefer the end network $g_{\text {assi,green }}$ to the current network.

Hence, $\phi(g) \cap\left\{g_{\text {assi,green }}\right\} \neq \emptyset$ for all $g \neq g_{\text {assi,green }}$ and $G=\left\{g_{\text {assi,green }}\right\}$ satisfies (ES).

Notice that if $n^{B} \leq n^{G} \leq n^{B}+1$, we can replicate the above proof (by just switching blue individuals for green ones and vice versa) to show that the set $G=\left\{g_{\text {assi,blue }}\right\}$ is a myopic-farsighted stable set. However, once $n^{G}>n^{B}+1$, the set $G=\left\{g_{\text {assi,blue }}\right\}$ is never a myopic-farsighted stable set because $\phi\left(g_{\text {seg }}\right) \cap\left\{g_{\text {assi,blue }}\right\}=\emptyset$. Moreover, the set $G=\left\{g_{\text {seg }}\right\}$ is never a myopic-farsighted stable set because $\phi\left(g_{\text {assi,green }}\right) \cap\left\{g_{\text {seg }}\right\}=\emptyset$. Thus, the complete segregation network $g_{\text {seg }}$ and the network $g_{\text {assi,blue }}$ in which all green individuals are fully assimilated into the smaller blue community are unlikely to emerge in the long run when all individuals are farsighted.

Remark 1. Assume low intra-community costs, $0<\Delta$ or $c<\delta-\delta^{2}$ and inter-community costs, $C>0$. Assume all individuals are farsighted, $N^{F}=N$.

(i) The set $G=\left\{g_{\text {assi,blue }}\right\}$ is never a myopic-farsighted stable set if $n^{G}>n^{B}+1$.

(ii) The set $G=\left\{g_{\text {seg }}\right\}$ is never a myopic-farsighted stable set. 


\section{Intermediate intra-community costs}

We now consider situations where intra-community costs are intermediate, i.e. $\delta-\delta^{2}<$ $c<\delta$. So, it becomes more expensive to build links with individuals from the same community. We denote by $g^{* i}$ the star network where individual $i$ is the center of the star.

Proposition 9. Assume intermediate intra-community costs, $\delta-\delta^{2}<c<\delta, N^{F} \neq \emptyset$ and $N^{M} \neq \emptyset$. If $\delta-\delta^{2}<c+C<\left(\delta-\delta^{2}\right)\left(1+\delta\left(n^{F}-1\right)\right)$, then the set $G^{*}=\left\{g^{* i} \mid i \in N^{M}\right\}$ is the unique myopic-farsighted stable set.

Proof. We first show that $G^{*}=\left\{g^{* i} \mid i \in N^{M}\right\}$ satisfies both internal stability (i.e. condition (IS) in Definition 3) and external stability (i.e. condition (ES) in Definition 3).

IS. Farsighted individuals are peripherals in all networks in $G^{*}$ so that they always obtain the same payoff: $U_{i}(g)=\delta+(n-2) \delta^{2}-c$ for all $i \in N^{F}, g \in G^{*}$. Myopic individuals who are peripherals have no incentive to delete their single link $\left(\delta+(n-2) \delta^{2}-c>0\right)$ nor to add a new link to any other individual $\left(2 \delta+(n-3) \delta^{2}-2 c<\delta+(n-2) \delta^{2}-c\right.$ since $\left.\delta-\delta^{2}<c\right)$. The center who is myopic has no incentive to delete one link since $c<\delta$. Hence, for every $g, g^{\prime} \in G^{*}$, it holds that $g^{\prime} \notin \phi(g)$.

ES. Take any network $g \notin G^{*}$. We build in steps a myopic-farsighted improving path from $g$ to some $g^{* i} \in G^{*}$.

Step 1: Starting in $g$, farsighted individuals delete all their links successively looking forward to some $g^{* i} \in G^{*}$, where they obtain their highest possible payoff given $\delta-\delta^{2}<c$. Notice that if $g$ is a star network where the center is a farsighted individual, then the center starts by deleting all her links since only the center is better off in $g^{* i}$ compared to $g$ (and we go directly to Step 8). We reach a network $g^{1}$ where all farsighted individuals have no link and myopic individuals only keep the links to myopic individuals they had in $g$.

Step 2: From $g^{1}$, looking forward to $g^{* i} \in G^{*}$, farsighted individuals build a star network $g^{* j F}$ restricted to farsighted individuals with individual $j$ being the center (i.e. $g^{* j F}$ is such that $j \in N^{F}, N_{j}\left(g^{* j F}\right)=N^{F} \backslash\{j\}$ and $N_{k}\left(g^{* j F}\right)=\{j\}$ for all $\left.k \in N^{F} \backslash\{j\}\right)$, and we obtain $g^{2}=g^{1} \cup g^{* j F}$ where all farsighted individuals are still disconnected from the myopic individuals.

Step 3: From $g^{2}$, looking forward to $g^{* i} \in G^{*}$, the farsighted individual $j$ who is the center of $g^{* j F}$ adds a link to some myopic individual, say individual 1 . Individual $j$ is better off in $g^{* i}$ compared to $g^{2}, \delta+(n-2) \delta^{2}-c>\left(n-n^{M}-1\right)(\delta-c)$, while individual 1 is better in $g^{2}+j 1$ if $c+C<\delta+\delta^{2}\left(n^{F}-1\right)$. This last inequality holds since $c+C<\left(\delta-\delta^{2}\right)\left(1+\delta\left(n^{F}-1\right)\right)<\delta+\delta^{2}\left(n^{F}-1\right)$.

Step 4: From $g^{2}+j 1$, looking forward to $g^{* i} \in G^{*}$, the farsighted individual $j$ adds a link successively to the myopic individuals who are neighbors of individual 1 (if any), say individual 2. Individual 2 who is myopic and linked to individual 1 has an incentive to 
add the link $j 2$ if $\delta^{2}+\left(n-n^{M}-1\right) \delta^{3}<\delta-c-C+\left(n-n^{M}-1\right) \delta^{2}$. Thus, a sufficient condition for adding the link is

$$
c+C<\delta-\delta^{2}+\left(n-n^{M}-1\right)\left(\delta^{2}-\delta^{3}\right),
$$

or

$$
c+C<\left(\delta-\delta^{2}\right)\left(1+\delta\left(n^{F}-1\right)\right)
$$

where $n-n^{M}$ is the number of farsighted individuals $\left(n^{F}\right)$. In the network $g^{2}+j 1+$ $\left\{j l \mid l \in N_{1}\left(g^{2}+j 1\right) \cap N^{M}\right\}$, individual $j$ is (directly) linked to all other farsighted individuals, individual 1 and all neighbors of individual 1.

Step 5: From $g^{2}+j 1+\left\{j l \mid l \in N_{1}\left(g^{2}+j 1\right) \cap N^{M}\right\}$, the myopic individuals who are neighbors of individual 1 and have just added a link to the farsighted individual $j$ delete their link successively with individual 1 . They have incentives to do so since $\delta-\delta^{2}<c<\delta$ and we reach $g^{2}+j 1+\left\{j l \mid l \in N_{1}\left(g^{2}+j 1\right) \cap N^{M}\right\}-\left\{1 l \mid l \in N_{1}\left(g^{2}+j 1\right) \cap N^{M}\right\}$.

Step 6: Next, looking forward to $g^{* i} \in G^{*}$, the farsighted individual $j$ adds a link successively to the myopic individuals who are neighbors of some $l \in N_{1}\left(g^{2}+j 1\right) \cap N^{M}$ and we proceed as in Step 4 and Step 5. We repeat this process until we reach a network $g^{3}$ where there is no myopic individual linked directly to the myopic neighbors of individual $j$ (i.e. $N_{k}\left(g^{3}\right) \cap N^{M}=\emptyset$ for all $k \in N_{j}\left(g^{3}\right) \cap N^{M}$ ).

Step 7: From $g^{3}$, individual $j$ adds a link to some myopic individual belonging to another component (if any) as in Step 3 and we proceed as in Step 4 to Step 6. We repeat this process until we end up with a star network $g^{* j}$ with individual $j$ (who is farsighted) in the center (i.e. $N_{j}\left(g^{* j}\right)=N \backslash\{j\}$ and $N_{k}\left(g^{* j}\right)=\{j\}$ for all $k \in N \backslash\{j\}$ ).

Step 8: From $g^{* j}$, looking forward to $g^{* i} \in G^{*}$, the farsighted individual $j$ deletes all her links successively to reach the empty network $g^{\emptyset}$. From $g^{\emptyset}$, myopic and farsighted individuals have both incentives ( network $g^{* i} \in G^{*}$ where some myopic individual $i \in N^{M}$ is the center.

We now show that $G^{*}$ is the unique myopic-farsighted stable set. Farsighted individuals who are peripherals in all networks in $G^{*}$ obtain their highest possible payoff. Myopic individuals who are peripherals have no incentive to delete their single link nor to add a new link. The center who is myopic has no incentive to delete one link. Hence, $\phi(g)=\emptyset$ for every $g \in G^{*}$. Suppose that $G \neq G^{*}$ is another myopic-farsighted stable set. (1) $G$ does not include $G^{*}: G \nsupseteq G^{*}$. External stability would be violated since $\phi(g)=\emptyset$ for every $g \in G^{*}$. (2) $G$ includes $G^{*}: G \nsupseteq G^{*}$. Internal stability would be violated since for every $g \in \mathcal{G} \backslash G^{*}$, it holds that $\phi(g) \cap G^{*} \neq \emptyset$.

Thus, when intra-community costs are intermediate and the population is formed by myopic and farsighted individuals, the set of star networks with a myopic individual at the center of the star is both a myopic-farsighted stable set and strongly efficient. From the proof of Proposition 9 we get the characterization of the myopic-farsightedly pairwise stable networks: if $\delta-\delta^{2}<c+C<\left(\delta-\delta^{2}\right)\left(1+\delta\left(n^{F}-1\right)\right)$, then $P_{M F}=\left\{g^{* i} \mid i \in N^{M}\right\}$. 
Once all individuals become farsighted (i.e. $N=N^{F}$ ), for $\delta-\delta^{2}<c<\delta$ and for $C>0$, every set consisting of a star network encompassing all individuals is a myopic-farsighted stable set

Proposition 10. Assume intermediate intra-community costs, $\delta-\delta^{2}<c<\delta$, and all individuals farsighted, $N=N^{F}$. If $g$ is a star network then $\{g\}$ is a myopic-farsighted stable set.

Proof. Since each set is a singleton set, internal stability (IS) is satisfied. (ES) Take any network $g \neq g^{* i}$, we need to show that $\phi(g) \ni g^{* i}$. (i) Suppose $g \neq g^{* j}(j \neq i)$. From $g$, looking forward to $g^{* i}$ (where they obtain their highest possible payoff), farsighted individuals $(\neq i)$ delete all their links successively to reach the empty network. From $g^{\emptyset}$, farsighted individuals have incentives (since $\delta>c$ ) to add links successively to build the star network $g^{* i}$ with individual $i$ in the center. (ii) Suppose $g=g^{* j}(j \neq i)$. From $g$, looking forward to $g^{* i}$, the farsighted individual $j$ deletes all her links successively to reach the empty network. From $g^{\emptyset}$, farsighted individuals have incentives (since $\delta>c$ ) to add links successively to build the star network $g^{* i}$ with individual $i$ in the center.

While every set consisting of a star network is a myopic-farsighted stable set, there may be other myopic-farsighted stable sets. Nevertheless, every star network encompassing all individuals is strongly efficient.

\section{Conclusion}

We have reconsidered de Marti and Zenou (2017) model of friendship network formation where individuals belong to two different communities (greens and blues). We have added a second heterogeneity dimension: individuals can be either myopic or farsighted. Our main results for low intra-community costs are summarized in Figure 8. When all individuals are myopic many friendship networks (complete integration, complete segregation, (partial) assimilation, ...) can be pairwise stable and a tension between efficiency and stability may occur. Once the population becomes mixed in terms of farsightedness and myopia, most inefficient friendship networks tend to be destabilized. When the larger (smaller) community is farsighted while the smaller (larger) community is myopic, the friendship network where the myopic community is assimilated into the farsighted community emerges in the long run when inter-community costs are large enough. Once all individuals are farsighted, the friendship network where the smaller community ends up being assimilated into the dominant community is likely to arise. When inter-community costs are small enough, the complete integration is stable whatever the number of farsighted and myopic individuals in both communities.

What would happen if there are farsighted and myopic individuals in both communities when intra-community costs are low and inter-community costs are large? Take the friendship network $g=\{12,13,14,23,24,34,56\}$ depicted in Figure 6 with $N^{F}=\{2,3,4\}$, 


\begin{tabular}{|c|c|c|}
\hline & Myopic & Farsighted \\
\hline Myopic & $\begin{array}{l}\text { Segregation } \\
\text { Assimilation to } \\
\text { Greens / Blues } \\
\text { Integration } \\
+ \text { many others }\end{array}$ & $\begin{array}{c}\text { Assimilation } \\
\text { to Greens } \\
\left(\text { for } C>\bar{C}_{1}\right) \\
\text { Integration } \\
\left(\text { for } n^{G} C<\Delta \text { ) }\right.\end{array}$ \\
\hline \multirow[t]{2}{*}{ Farsighted } & $\begin{array}{l}\text { Assimilation } \\
\text { to Blues } \\
\text { (for } C>\bar{C}_{2} \text { ) }\end{array}$ & $\begin{array}{l}\text { Assimilation } \\
\text { to Greens } \\
\text { (for } C>0 \text { ) }\end{array}$ \\
\hline & $\begin{array}{c}\text { Integration } \\
\left(\text { for } n^{G} C<\Delta\right)\end{array}$ & $\begin{array}{c}\text { Integration } \\
\text { (for } n^{G} C<\Delta \text { ) }\end{array}$ \\
\hline
\end{tabular}

Figure 8: A summary of stable friendship networks with low intra-community costs.

$N^{M}=\{1,5,6\}, N^{G}=\{1,2,3\}$ and $N^{B}=\{4,5,6\}$. There are no myopic-farsighted improving paths emanating from $g$ when inter-community costs are large; $\phi(g)=\emptyset$. Hence, this friendship network, where the farsighted blue individual is assimilated to the dominant green community, belongs to all myopic-farsighted stable sets (if they exist). ${ }^{13}$ However, the complete segregated network $g^{\prime}=\{12,13,23,45,46,56\}$ will never occur since $g \in \phi\left(g^{\prime}\right)$ and $G \supseteq\left\{g, g^{\prime}\right\}$ would violate internal stability. Indeed, the farsighted blue individual 4 has incentives to first delete her links in $g^{\prime}$ and next build the links with all green individuals to form $g$. Providing a full-fledged characterization of the myopic-farsighted stable sets turns to be extremely hard, if not impossible. To summarize, depending on the costs for interacting, either a fully integrated society or a (partially) assimilated society are likely to arise in the long run. In addition, farsightedness seems to dampen the tension between efficiency and stability in friendship networks when individuals belong to different communities.

Notice that the degree of farsightedness of an individual is likely to be correlated with her level of education or grades at school. Hence, for future research it would be interesting to confront our theoretical predictions with data. (i) In presence of only high educated communities, is it likely that the smaller community ends up assimilated into the dominant one? (ii) In presence of a high educated community and a low educated community, is it likely that the lower educated community ends up assimilated into the high educated one? (iii) Complete segregation mostly occurs when both communities are low educated. (iv) When one community has a large number of high educated individuals while the other community has a low number of high educated individuals, is it likely that the high educated individuals belonging to the less educated community end up

\footnotetext{
${ }^{13}$ Patacchini and Zenou (2016) look at friendship networks among US high-school students (Add Health data). They find that, for mixed schools, most of the white students have white friends while one part of the black students has mostly white friends and the other part has mostly black friends.
} 
assimilated into the more educated community?

\section{Acknowledgements}

Ana Mauleon and Vincent Vannetelbosch are, respectively, Research Director and Senior Research Associate of the National Fund for Scientific Research (FNRS). Financial support from the MSCA ITN Expectations and Social Influence Dynamics in Economics (ExSIDE) Grant No721846 (1/9/2017-31/8/2020), from the Belgian French speaking community ARC project 15/20- 072 of Saint-Louis University - Brussels, and from the Fonds de la Recherche Scientifique - FNRS research grant T.0143.18 is gratefully acknowledged.

\section{Appendix}

\section{A More pairwise stable friendship networks}

We now show that any network where $n^{G}$ green individuals are fully intra-connected, $n^{B}$ blue individuals are fully intra-connected, and one green individual is linked to all blue individuals is pairwise stable for intermediate inter-community costs. In Figure 9 we depict such a network.

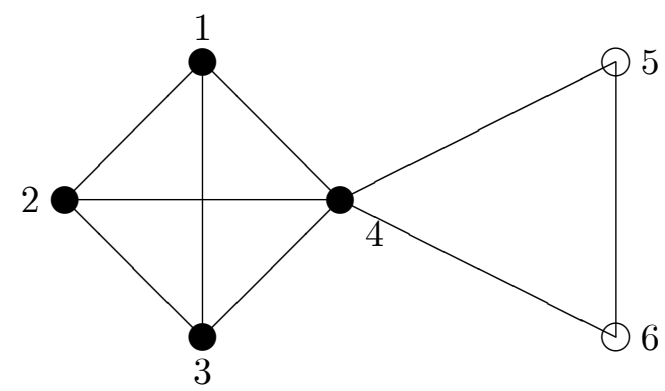

Figure 9: Both communities are fully intra-connected and one green individual is linked to all blue individuals.

Proposition 11. Assume low intra-community costs, $0<\Delta$ or $c<\delta-\delta^{2}$. The network $\widetilde{g}=g^{N^{G}} \cup g^{N^{B}} \cup\left\{i j \mid j \in N^{B}, i=\widetilde{i}\right.$ with $\left.\widetilde{i} \in N^{G}\right\}$ where $n^{G}$ green individuals are fully intra-connected, $n^{B}$ blue individuals are fully intra-connected, and one green individual is linked to all blue individuals is pairwise stable if and only if

$$
\underline{C}_{3}<C<\bar{C}_{3}
$$


where

$$
\begin{aligned}
& \underline{C}_{3}=\frac{n^{B}}{n^{B}-1} \Delta \\
& \bar{C}_{3}=\min \left\{\frac{(n-2)(n-3)}{\left(n^{G}-1\right)\left(n^{G}-2\right)} \Delta, \frac{(n-2)(n-3)}{\left(n^{B}\right)\left(n^{B}-1\right)} \Delta, \frac{(n-2)}{\left(n^{G}-1\right)}\left(\Delta+\delta^{2}(1-\delta)\left(n^{G}-1\right)\right)\right\} .
\end{aligned}
$$

Proof. Individual $\widetilde{i} \in N^{G}$ is the green individual who is linked to all individuals in $\widetilde{g}$.

(i) In $\widetilde{g}$ individual $\widetilde{i}$ has no incentive to cut a link with a blue individual if and only if

$$
C<\frac{(n-2)(n-3)}{\left(n^{G}-1\right)\left(n^{G}-2\right)} \Delta .
$$

In $\widetilde{g}$ any blue individual has no incentive to cut a link with the green individual $\widetilde{i}$ if and only if

$$
C<\frac{(n-2)}{\left(n^{G}-1\right)}\left(\Delta+\delta^{2}(1-\delta)\left(n^{G}-1\right)\right) .
$$

Combining these two conditions, a link between $\widetilde{i}$ and a blue individual will not be deleted if and only if

$$
C<\min \left\{\frac{(n-2)(n-3)}{\left(n^{G}-1\right)\left(n^{G}-2\right)} \Delta, \frac{(n-2)}{\left(n^{G}-1\right)}\left(\Delta+\delta^{2}(1-\delta)\left(n^{G}-1\right)\right)\right\} .
$$

(ii) In $\widetilde{g}$ player $\widetilde{i}$ has no incentive to cut a link with a green individual if and only if

$$
C<\frac{(n-2)(n-3)}{\left(n^{B}\right)\left(n^{B}-1\right)} \Delta .
$$

Since $\Delta+n^{B} \delta^{2}(1-\delta)>0$, any green individual $i \neq \widetilde{i}$ has no incentive to delete her link with $\widetilde{i}$. Moreover, since $0<\Delta$, any green individual $i \neq \widetilde{i}$ has no incentive to delete her link with another green individual $j \neq \widetilde{i}$. Thus, a link between any two green individuals will not be deleted if and only if

$$
C<\frac{(n-2)(n-3)}{\left(n^{B}\right)\left(n^{B}-1\right)} \Delta .
$$

(iii) Since $0<\Delta$, any blue individual has no incentive to delete her link with another blue individual.

(iv) In $\widetilde{g}$ any green individual $i \neq \widetilde{i}$ has no incentive to add a link with a blue individual if and only if

$$
C>\frac{n^{B}}{n^{B}-1} \Delta
$$

In addition, any blue individual has no incentive to add a link to a green individual $i \neq \widetilde{i}$ if and only if

$$
C>\frac{n^{B}(n-2)}{\left(n^{B}-1\right)(n-2)-\left(n^{G}-1\right)} \Delta .
$$


Combining these two conditions, a link between any green individual $i \neq \widetilde{i}$ and a blue individual will not be added if and only if

$$
C>\frac{n^{B}}{n^{B}-1} \Delta=\underline{C}_{3}
$$

From (i), (ii), (iii) and (iv) we have that $\widetilde{g}$ is pairwise stable if and only if $\underline{C}_{3}<C<$ $\bar{C}_{3}$, where

$$
\bar{C}_{3}=\min \left\{\frac{(n-2)(n-3)}{\left(n^{G}-1\right)\left(n^{G}-2\right)} \Delta, \frac{(n-2)(n-3)}{\left(n^{B}\right)\left(n^{B}-1\right)} \Delta, \frac{(n-2)}{\left(n^{G}-1\right)}\left(\Delta+\delta^{2}(1-\delta)\left(n^{G}-1\right)\right)\right\} .
$$

We now show that any network where both communities are fully intra-connected, some blue individuals are assimilated to the green community and some green individuals are assimilated to the blue community is pairwise stable for intermediate inter-community costs. In Figure 10 we depict such a network.

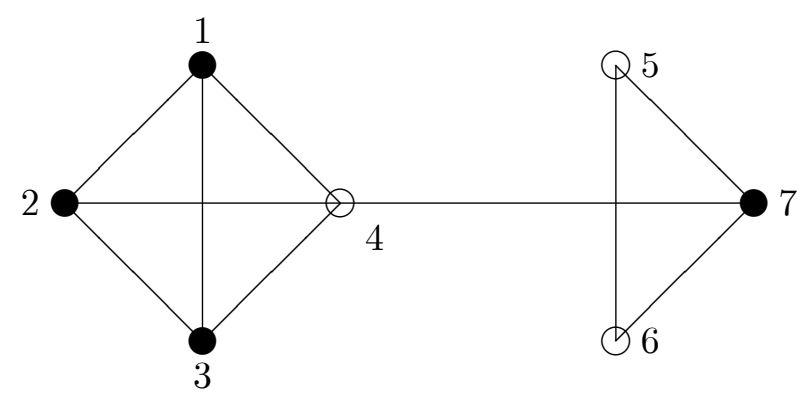

Figure 10: One green and one blue individual are assimilated to the other community.

Proposition 12. Assume low intra-community costs, $0<\Delta$ or $c<\delta-\delta^{2}$. The network $\widehat{g}=g^{N^{G} \backslash N^{G_{1}}} \cup g^{N^{B} \backslash N^{B_{1}}} \cup\left\{i j \mid i \in N^{B_{1}}, j \in N^{G}\right\} \cup\left\{i j \mid i \in N^{G_{1}}, j \in N^{B}\right\}$, where both communities are fully intra-connected, $n^{B_{1}}\left(1 \leq n^{B_{1}}<n^{B}\right)$ blue individuals are assimilated to the green community and $n^{G_{1}}\left(1 \leq n^{G_{1}}<n^{G}\right)$ green individuals are assimilated to the blue community, is pairwise stable if and only if

$$
\begin{aligned}
C> & \max \left\{\frac{n^{B}\left(n^{B}+n^{G_{1}}-n^{B_{1}}-2\right)}{\left(n^{B}-n^{B_{1}}\right)\left(n^{B}-n^{B_{1}}-1\right)} \Delta, \frac{n^{G}\left(n^{G}+n^{B_{1}}-n^{G_{1}}-2\right)}{\left(n^{G}-n^{G_{1}}\right)\left(n^{G}-n^{G_{1}}-1\right)} \Delta,\right. \\
& \left.\frac{\left(n^{G}+n^{B_{1}}-n^{G_{1}}-1\right)\left(n^{B}+n^{G_{1}}-n^{B_{1}}-1\right)}{\left(n^{G}-n^{G_{1}}-1\right)\left(n^{B}-n^{B_{1}}-1\right)}\left(\Delta+\delta^{2}(1-\delta)\left(n^{B}-n^{B_{1}}\right)\right)\right\} .
\end{aligned}
$$

Proof. In $\widehat{g}$ the $n^{B_{1}}\left(1 \leq n^{B_{1}}<n^{B}\right)$ blue individuals are fully assimilated to the green community (with payoff $\left.n^{G}(\delta-c)\right)$ and the $n^{G_{1}}\left(1 \leq n^{G_{1}}<n^{G}\right)$ green individuals are fully assimilated to the blue community (with payoff $n^{B}(\delta-c)$ ), while the other $n^{B}-n^{B_{1}}$ blue 
individuals obtain $\left(n^{B}-n^{B_{1}}+n^{G_{1}}-1\right)(\delta-c)$ and the other $n^{G}-n^{G_{1}}$ green individuals obtain $\left(n^{G}-n^{G_{1}}+n^{B_{1}}-1\right)(\delta-c)$. Since $0<\Delta$ or $c<\delta-\delta^{2}$, all individuals have no incentive to delete a link in $\widehat{g}$.

(i) In $\widehat{g}$ any green individual $i \in N^{G_{1}}$ has no incentive to add a link to another green individual $j \in N^{G} \backslash N^{G_{1}}$ if and only if

$$
C>\frac{n^{B}\left(n^{B}+n^{G_{1}}-n^{B_{1}}-2\right)}{\left(n^{B}-n^{B_{1}}\right)\left(n^{B}-n^{B_{1}}-1\right)} \Delta .
$$

Since $\Delta+\delta^{2}(1-\delta)\left(n^{B}-n^{B_{1}}\right)>0$, any green individual $j \in N^{G} \backslash N^{G_{1}}$ has always incentives to form a link with a green individual $i \in N^{G_{1}}$. Hence, a link between a green individual $i \in N^{G_{1}}$ and a green individual $j \in N^{G} \backslash N^{G_{1}}$ will not be formed in $\widehat{g}$ if and only if

$$
C>\frac{n^{B}\left(n^{B}+n^{G_{1}}-n^{B_{1}}-2\right)}{\left(n^{B}-n^{B_{1}}\right)\left(n^{B}-n^{B_{1}}-1\right)} \Delta .
$$

(ii) In $\widehat{g}$ any blue individual $i \in N^{B_{1}}$ has no incentive to add a link to a blue individual $j \in N^{B} \backslash N^{B_{1}}$ if and only if

$$
\frac{n^{G}\left(n^{G}+n^{B_{1}}-n^{G_{1}}-2\right)}{\left(n^{G}-n^{G_{1}}\right)\left(n^{G}-n^{G_{1}}-1\right)} \Delta .
$$

Since $\Delta+\delta^{2}(1-\delta)\left(n^{G}-n^{G_{1}}\right)>0$, any blue individual $j \in N^{B} \backslash N^{B_{1}}$ has always incentives to form a link with a blue individual $i \in N^{B_{1}}$. Hence, a link between a blue individual $i \in N^{B_{1}}$ and a blue individual $j \in N^{B} \backslash N^{B_{1}}$ will not be formed in $\widehat{g}$ if and only if

$$
\frac{n^{G}\left(n^{G}+n^{B_{1}}-n^{G_{1}}-2\right)}{\left(n^{G}-n^{G_{1}}\right)\left(n^{G}-n^{G_{1}}-1\right)} \Delta .
$$

(iii) In $\widehat{g}$ any blue individual $i \in N^{B_{1}}$ has no incentive to add a link to another blue individual $j \in N^{B_{1}}$ if and only if

$$
C>\frac{n^{G}\left(n^{G}+n^{B_{1}}-n^{G_{1}}-2\right)}{\left(n^{G}-n^{G_{1}}\right)\left(n^{G}-n^{G_{1}}-1\right)} \Delta .
$$

(iv) In $\widehat{g}$ any green individual $i \in N^{G_{1}}$ has no incentive to add a link to another green individual $j \in N^{G_{1}}$ if and only if

$$
C>\frac{n^{B}\left(n^{B}+n^{G_{1}}-n^{B_{1}}-2\right)}{\left(n^{B}-n^{B_{1}}\right)\left(n^{B}-n^{B_{1}}-1\right)} \Delta .
$$

(v) In $\widehat{g}$ any green individual $i \in N^{G} \backslash N^{G_{1}}$ has no incentive to add a link to a blue individual $j \in N^{B} \backslash N^{B_{1}}$ if and only if

$$
C>\frac{\left(n^{G}+n^{B_{1}}-n^{G_{1}}-1\right)\left(n^{B}+n^{G_{1}}-n^{B_{1}}-1\right)}{\left(n^{G}-n^{G_{1}}-1\right)\left(n^{B}-n^{B_{1}}-1\right)}\left(\Delta+\delta^{2}(1-\delta)\left(n^{B}-n^{B_{1}}\right)\right) .
$$

In $\widehat{g}$ any blue individual $j \in N^{B} \backslash N^{B_{1}}$ has no incentive to add a link to a green individual $i \in N^{G} \backslash N^{G_{1}}$ if and only if

$$
C>\frac{\left(n^{G}+n^{B_{1}}-n^{G_{1}}-1\right)\left(n^{B}+n^{G_{1}}-n^{B_{1}}-1\right)}{\left(n^{G}-n^{G_{1}}-1\right)\left(n^{B}-n^{B_{1}}-1\right)}\left(\Delta+\delta^{2}(1-\delta)\left(n^{G}-n^{G_{1}}\right)\right) .
$$


Hence, a link between a green individual $i \in N^{G} \backslash N^{G_{1}}$ and a blue individual $j \in N^{B} \backslash N^{B_{1}}$ will not be formed in $\widehat{g}$ if and only if

$$
\begin{aligned}
C> & \min \left\{\frac{\left(n^{G}+n^{B_{1}}-n^{G_{1}}-1\right)\left(n^{B}+n^{G_{1}}-n^{B_{1}}-1\right)}{\left(n^{G}-n^{G_{1}}-1\right)\left(n^{B}-n^{B_{1}}-1\right)}\left(\Delta+\delta^{2}(1-\delta)\left(n^{B}-n^{B_{1}}\right)\right),\right. \\
& \left.\frac{\left(n^{G}+n^{B_{1}}-n^{G_{1}}-1\right)\left(n^{B}+n^{G_{1}}-n^{B_{1}}-1\right)}{\left(n^{G}-n^{G_{1}}-1\right)\left(n^{B}-n^{B_{1}}-1\right)}\left(\Delta+\delta^{2}(1-\delta)\left(n^{G}-n^{G_{1}}\right)\right)\right\} .
\end{aligned}
$$

From (i), (ii), (iii), (iv) and (v) we have that the network $\widehat{g}$ is pairwise stable if and only if

$$
\begin{aligned}
C> & \max \left\{\frac{n^{B}\left(n^{B}+n^{G_{1}}-n^{B_{1}}-2\right)}{\left(n^{B}-n^{B_{1}}\right)\left(n^{B}-n^{B_{1}}-1\right)} \Delta, \frac{n^{G}\left(n^{G}+n^{B_{1}}-n^{G_{1}}-2\right)}{\left(n^{G}-n^{G_{1}}\right)\left(n^{G}-n^{G_{1}}-1\right)} \Delta,\right. \\
& \left.\frac{\left(n^{G}+n^{B_{1}}-n^{G_{1}}-1\right)\left(n^{B}+n^{G_{1}}-n^{B_{1}}-1\right)}{\left(n^{G}-n^{G_{1}}-1\right)\left(n^{B}-n^{B_{1}}-1\right)}\left(\Delta+\delta^{2}(1-\delta)\left(n^{B}-n^{B_{1}}\right)\right)\right\} .
\end{aligned}
$$

\section{B Proofs}

Proof of Proposition 2. Assume low intra-community costs, $0<\Delta$ or $c<\delta-\delta^{2}$.

(i) We show that the network $g_{\text {assi,green }}=g^{N^{G}} \cup\left\{i j \mid i \in N^{G}, j \in N^{B}\right\}$ where all the blue community is fully assimilated to the green community is pairwise stable if and only if

$$
C>\frac{(n-2)}{\left(n^{G}-1\right)} \Delta
$$

In $g_{\text {assi,green }}$ all green individuals get as payoff $(n-1)(\delta-c)$ and all blue individuals get as payoff $\left(n^{G}\right)(\delta-c)$. Since $0<\Delta$, all blue individuals have no incentive to cut a link and all green individuals have no incentive to cut a link with a green or blue individual. In $g_{\text {assi,green }}$, any blue individual will not add a link to another blue individual if and only

$$
C>\frac{(n-2)}{\left(n^{G}-1\right)} \Delta .
$$

(ii) We show that the network $g_{\text {assi,blue }}=g^{N^{B}} \cup\left\{i j \mid i \in N^{B}, j \in N^{G}\right\}$ where all the green community is fully assimilated to the blue community is pairwise stable if and only if

$$
C>\frac{(n-2)}{\left(n^{B}-1\right)} \Delta
$$

In $g_{\text {assi,blue }}$ all blue individuals get as payoff $(n-1)(\delta-c)$ and all green individuals get as payoff $\left(n^{B}\right)(\delta-c)$. Since $0<\Delta$, all green individuals have no incentive to cut a link and all blue individuals have no incentive to cut a link with a green or blue individual. In $g_{\text {assi,blue }}$, any green individual will not add a link to another green individual if and only

$$
C>\frac{(n-2)}{\left(n^{B}-1\right)} \Delta
$$


(iii) Take any $N^{B_{1}} \varsubsetneqq N^{B}$ such that $1 \leq n^{B_{1}} \leq n^{B}-2$. We show that the network $g_{\text {passi,green }}=g^{N^{G}} \cup g^{N^{B} \backslash N^{B_{1}}} \cup\left\{i j \mid i \in N^{G}, j \in N^{B_{1}}\right\}$ where $n^{B_{1}}$ blue individuals are assimilated to the green individuals and all other blue individuals are intra-connected and segregated is pairwise stable if and only if

$$
C>\frac{\left(n^{G}+n^{B_{1}}-1\right)}{\left(n^{G}-1\right)}\left(\Delta+\left(n^{B}-n^{B_{1}}\right) \delta^{2}\right) .
$$

(iiia) In $g_{\text {passi,green }}$, all segregated blue individuals get as payoff $\left(n^{B}-n^{B_{1}}-1\right)(\delta-c)$, all assimilated blue individuals get as payoff $\left(n^{G}\right)(\delta-c)$ and all green individuals get as payoff $\left(n^{G}+n^{B_{1}}-1\right)(\delta-c)$. Since $0<\Delta$, all individuals have no incentive to cut a link. In $g_{\text {passi,green }}$, any green individual will not add a link to a blue individual $j \in N^{B} \backslash N^{B_{1}}$ if and only if

$$
C>\frac{\left(n^{G}+n^{B_{1}}-1\right)}{\left(n^{G}-1\right)}\left(\Delta+\left(n^{B}-n^{B_{1}}\right) \delta^{2}\right) .
$$

In $g_{\text {passi,green }}$, any blue individual $j \in N^{B} \backslash N^{B_{1}}$ will not add a link to a green individual if and only if

$$
C>\frac{\left(n^{G}+n^{B_{1}}-1\right)}{\left(n^{G}-1\right)}\left(\Delta+\left(n^{G}+n^{B_{1}}\right) \delta^{2}\right) .
$$

Hence, by mutual consent, a link between a blue individual $j \in N^{B} \backslash N^{B_{1}}$ and a green individual will not be added in $g_{\text {passi,green }}$ if and only if

$$
\begin{aligned}
C & >\min \left\{\frac{\left(n^{G}+n^{B_{1}}-1\right)}{\left(n^{G}-1\right)}\left(\Delta+\left(n^{G}+n^{B_{1}}\right) \delta^{2}\right), \frac{\left(n^{G}+n^{B_{1}}-1\right)}{\left(n^{G}-1\right)}\left(\Delta+\left(n^{B}-n^{B_{1}}\right) \delta^{2}\right)\right\} \\
& =\frac{\left(n^{G}+n^{B_{1}}-1\right)}{\left(n^{G}-1\right)}\left(\Delta+\left(n^{B}-n^{B_{1}}\right) \delta^{2}\right) .
\end{aligned}
$$

(iiib) In $g_{\text {passi,green }}$, any blue individual $i \in N^{B_{1}}$ will not add a link to a blue individual $j \in N^{B} \backslash N^{B_{1}}$ if and only if

$$
C>\frac{\left(n^{G}+n^{B_{1}}-2\right)}{\left(n^{G}-1\right)}\left(\Delta+\left(n^{B}-n^{B_{1}}\right) \delta^{2}\right) .
$$

However, any blue individual $j \in N^{B} \backslash N^{B_{1}}$ has always incentives to add a link to a blue individual $i \in N^{B_{1}}$ since $0<\Delta$. Hence, a link between a blue individual $i \in N^{B_{1}}$ and a blue individual $j \in N^{B} \backslash N^{B_{1}}$ will not be added in $g_{\text {passi,green }}$ if and only if

$$
C>\frac{\left(n^{G}+n^{B_{1}}-2\right)}{\left(n^{G}-1\right)}\left(\Delta+\left(n^{B}-n^{B_{1}}\right) \delta^{2}\right) .
$$

(iiic) In $g_{\text {passi,green }}$, any blue individual $i \in N^{B_{1}}$ will not add a link to another blue individual $j \in N^{B_{1}}$ if and only if

$$
C>\frac{\left(n^{G}+n^{B_{1}}-2\right)}{\left(n^{G}-1\right)} \Delta .
$$


From (iiia), (iiib) and (iiic), we have that $g_{\text {passi,green }}$ (with $1 \leq n^{B_{1}} \leq n^{B}-2$ ) is pairwise stable if and only if

$$
C>\frac{\left(n^{G}+n^{B_{1}}-1\right)}{\left(n^{G}-1\right)}\left(\Delta+\left(n^{B}-n^{B_{1}}\right) \delta^{2}\right) .
$$

(iv) Take any $N^{G_{1}} \varsubsetneqq N^{G}$ such that $1 \leq n^{G_{1}} \leq n^{G}-2$. We show that the network $g_{\text {passi,blue }}=g^{N^{B}} \cup g^{N^{G} \backslash N^{G_{1}}} \cup\left\{i j \mid i \in N^{B}, j \in N^{G_{1}}\right\}$ where $n^{G_{1}}$ green individuals are assimilated to the blue individuals and all other green individuals are intra-connected and segregated is pairwise stable if and only if

$$
C> \begin{cases}\widehat{C}_{1} & \text { if } n^{G_{1}} \leq \frac{1}{2}\left(n^{G}-n^{B}\right) ; \\ \widehat{C}_{2} & \text { if } n^{G_{1}}>\frac{1}{2}\left(n^{G}-n^{B}\right) ;\end{cases}
$$

where

$$
\begin{aligned}
& \widehat{C}_{1}=\max \left\{\frac{\left(n^{B}+n^{G_{1}}-1\right)}{\left(n^{B}-1\right)}\left(\Delta+\left(n^{B}+n^{G_{1}}\right) \delta^{2}\right), \frac{\left(n^{B}+n^{G_{1}}-2\right)}{\left(n^{B}-1\right)}\left(\Delta+\left(n^{G}-n^{G_{1}}\right) \delta^{2}\right)\right\} ; \\
& \widehat{C}_{2}=\frac{\left(n^{B}+n^{G_{1}}-1\right)}{\left(n^{B}-1\right)}\left(\Delta+\left(n^{G}-n^{G_{1}}\right) \delta^{2}\right) .
\end{aligned}
$$

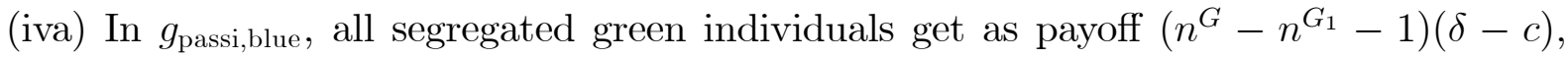
all assimilated green individuals get as payoff $\left(n^{B}\right)(\delta-c)$ and all blue individuals get as payoff $\left(n^{B}+n^{G_{1}}-1\right)(\delta-c)$. Since $0<\Delta$, all individuals have no incentive to cut a link. In $g_{\text {passi,blue }}$, any blue individual will not add a link to a green individual $j \in N^{G} \backslash N^{G_{1}}$ if and only if

$$
C>\frac{\left(n^{B}+n^{G_{1}}-1\right)}{\left(n^{B}-1\right)}\left(\Delta+\left(n^{G}-n^{G_{1}}\right) \delta^{2}\right) .
$$

In $g_{\text {passi,blue }}$, any green individual $j \in N^{G} \backslash N^{G_{1}}$ will not add a link to a blue individual if and only if

$$
C>\frac{\left(n^{B}+n^{G_{1}}-1\right)}{\left(n^{B}-1\right)}\left(\Delta+\left(n^{B}+n^{G_{1}}\right) \delta^{2}\right) .
$$

Hence, by mutual consent, a link between a green individual $j \in N^{G} \backslash N^{G_{1}}$ and a blue individual will not be added in $g_{\text {passi,blue }}$ if and only if

$$
C>\min \left\{\frac{\left(n^{B}+n^{G_{1}}-1\right)}{\left(n^{B}-1\right)}\left(\Delta+\left(n^{B}+n^{G_{1}}\right) \delta^{2}\right), \frac{\left(n^{B}+n^{G_{1}}-1\right)}{\left(n^{B}-1\right)}\left(\Delta+\left(n^{G}-n^{G_{1}}\right) \delta^{2}\right)\right\} .
$$

(ivb) In $g_{\text {passi,blue }}$, any green individual $i \in N^{G_{1}}$ will not add a link to another green individual $j \in N^{G} \backslash N^{G_{1}}$ if and only if

$$
C>\frac{\left(n^{B}+n^{G_{1}}-2\right)}{\left(n^{B}-1\right)}\left(\Delta+\left(n^{G}-n^{G_{1}}\right) \delta^{2}\right) .
$$


However, any green individual $j \in N^{G} \backslash N^{G_{1}}$ has always incentives to add a link to a green individual $i \in N^{G_{1}}$ since $0<\Delta$. Hence, a link between a green individual $i \in N^{G_{1}}$ and a green individual $j \in N^{G} \backslash N^{G_{1}}$ will not be added in $g_{\text {passi,blue }}$ if and only if

$$
C>\frac{\left(n^{B}+n^{G_{1}}-2\right)}{\left(n^{B}-1\right)}\left(\Delta+\left(n^{G}-n^{G_{1}}\right) \delta^{2}\right) .
$$

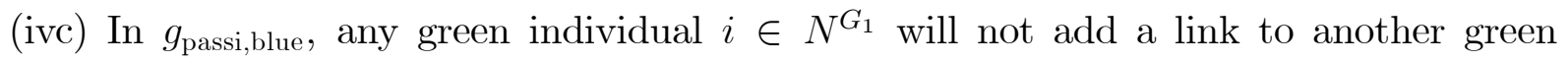
individual $j \in N^{G_{1}}$ if and only if

$$
C>\frac{\left(n^{B}+n^{G_{1}}-2\right)}{\left(n^{B}-1\right)} \Delta .
$$

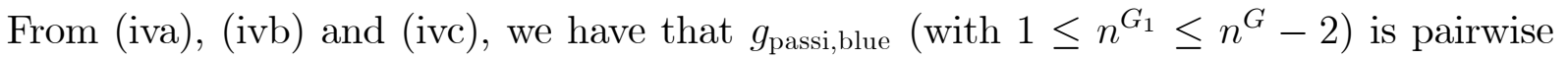
stable if and only if

$$
C> \begin{cases}\widehat{C}_{1} & \text { if } n^{G_{1}} \leq \frac{1}{2}\left(n^{G}-n^{B}\right) \\ \widehat{C}_{2} & \text { if } n^{G_{1}}>\frac{1}{2}\left(n^{G}-n^{B}\right) .\end{cases}
$$

Proof of Proposition 3. Assume low intra-community costs, $0<\Delta$ or $c<\delta-\delta^{2}$. In the complete segregated network $g_{\text {seg }}$, a green individual obtains $\left(n^{G}-1\right)(\delta-c)$ as payoff, while a blue obtains $\left(n^{B}-1\right)(\delta-c)$ as payoff. In the complete integrated network $g_{\text {int }}$, a green individual and a blue individual obtain, respectively,

$$
(n-1)(\delta-c)-n^{B} \frac{n^{G}-1}{n-2} \frac{n^{B}-1}{n-2} C \text { and }(n-1)(\delta-c)-n^{G} \frac{n^{B}-1}{n-2} \frac{n^{G}-1}{n-2} C
$$

as payoff. In the network where the blue individuals are fully assimilated to the green community $g_{\text {assi,green }}$, a green individual obtains $(n-1)(\delta-c)$ as payoff, while a blue obtains $\left(n^{G}\right)(\delta-c)+\left(n^{B}-1\right) \delta^{2}$ as payoff. In the network where the green individuals are fully assimilated to the blue community $g_{\text {assi,blue }}$, a blue individual obtains $(n-1)(\delta-c)$ as payoff, while a green obtains $\left(n^{B}\right)(\delta-c)+\left(n^{G}-1\right) \delta^{2}$ as payoff. Since, $n^{G} \geq n^{B}$, the network $g_{\text {assi,blue }}$ is never better than the network $g_{\text {assi,green }}$ in terms of strong efficiency. Comparing the network $g_{\text {assi,green }}$ with the complete integrated network $g_{\text {int }}$, we have that the complete integrated network $g_{\text {int }}$ is better than the network $g_{\text {assi,green }}$ in terms of strong efficiency (i.e. sum of the payoffs of all individuals) if and only if

$$
C<\frac{(n-2)^{2}}{2 n^{G}\left(n^{G}-1\right)} \Delta=C^{*} .
$$

In addition, we have that the network $g_{\text {assi,green }}$ is always better than the complete segregated network $g_{\text {seg }}$ in terms of strong efficiency: $n^{G}(n-1)(\delta-c)+n^{B}\left(n^{G}\right)(\delta-c)+$ $n^{B}\left(n^{B}-1\right) \delta^{2}>n^{G}\left(n^{G}-1\right)(\delta-c)+n^{B}\left(n^{B}-1\right)(\delta-c)$. 


\section{Proof of Proposition 4.}

In a star network, the center gets $(n-1)(\delta-c)$ as payoff while the individuals at the periphery get $\delta+(n-2) \delta^{2}-c$ as payoff. Since $\delta-\delta^{2}<c<\delta$, individuals at the periphery of a star network get their highest possible payoff. Hence, they will not add a link between them nor they will cut a link with the center. Obviously, the center has no incentive to cut a link to a peripheral individual. Thus, any star network is pairwise stable. Jackson and Wolinsky (1996) show that a star network is strongly efficient for $\delta-\delta^{2}<c<\delta$ (and $C=0)$. Hence, such star network is also strongly efficient for $\delta-\delta^{2}<c<\delta$ and $C>0$.

Proof of Proposition 7. The set $G=\left\{g_{\text {assi,blue }}\right\}$ satisfies (IS) in Definition 3 since it is a singleton set. We now show that it also satisfies (ES).

ES. Take any network $g \neq g_{\text {assi,blue }}$. We build in steps a myopic-farsighted improving path from $g$ to $g_{\text {assi,blue. }}$.

Step 0: If $g$ is such that $g \cap g^{N^{G}} \neq \emptyset$ then go to Step 1. Otherwise, starting from $g$, blue individuals first build all the missing links between blue individuals to reach $g^{\prime}=g \cup g^{N^{B}}$ looking forward to $g_{\text {assi,blue }}$, where they obtain their highest possible payoff given $c<\delta-\delta^{2}$, $U_{i}\left(g_{\text {assi,blue }}\right)=(n-1)(\delta-c)$. From $g^{\prime}$ blue individuals build all the missing links with green individuals to finally reach $g^{\prime \prime}=g^{\prime} \cup\left\{i j \mid i \in N^{B}, j \in N^{G}\right\}=g_{\text {assi,blue. Since }}$ $c<\delta-\delta^{2}$ and $g^{\prime \prime} \cap g^{N^{G}}=\emptyset$, green individuals are assimilated to the blue community in $g^{\prime \prime}$ and they are not affected by $C$ and so they have incentives to add the links with the blue individuals.

Step 1: Starting in $g$, blue individuals who are all farsighted $\left(N^{F}=N^{B}\right)$ delete successively all the links (if any) they have with blue individuals looking forward to $g_{\text {assi,blue }}$, where they obtain their highest possible payoff given $c<\delta-\delta^{2}, U_{i}\left(g_{\text {assi,blue }}\right)=(n-1)(\delta-c)$. We reach the network $g^{\prime}=g \backslash g^{N^{B}}$ where there are no links between blue individuals.

Step 2: From $g^{\prime}=g \backslash g^{N^{B}}$, since $c<\delta-\delta^{2}$, green individuals who are all myopic have incentives to build all the links with the blue individuals. Blue individuals who are looking forward $g_{\text {assi,blue }}$ prefer the end network to the current one. We reach the network $g^{\prime \prime}=g^{\prime} \cup\left\{i j \mid i \in N^{B}, j \in N^{G}\right\}$ where all possible links between green and blue individuals are formed.

Step 3: From $g^{\prime \prime}=g^{\prime} \cup\left\{i j \mid i \in N^{B}, j \in N^{G}\right\}$, since $c<\delta-\delta^{2}$, green individuals who are all myopic have incentives to build all the missing links between the green individuals. We reach the network $g^{\prime \prime \prime}=g^{\prime \prime} \cup g^{N^{G}}$ where all the blue individuals are assimilated to the green community and the green community is fully intra-connected. In fact, $g^{\prime \prime \prime}=g_{\text {assi,green }}$ and all blue individuals prefer $g_{\text {assi,blue }}$ to $g_{\text {assi,green }}$.

Step 4: From $g^{\prime \prime \prime}=g^{\prime \prime} \cup g^{N^{G}}$, blue individuals who are all farsighted and look forward towards $g_{\text {assi,blue }}$ build all the links between the blue individuals to reach $g^{N}$. 
Step 5: From the complete network $g^{N}$, since $C>\bar{C}_{2}$, green individuals who are myopic have incentives to delete successively all the links they have with other green individuals to finally reach the network $g_{\text {assi,blue }}=g^{N} \backslash g^{N^{G}}$. The condition $C>\bar{C}_{2}$ guarantees that, along the myopic-farsighted improving path starting at $g_{1}=g^{N}$, followed by $g_{k+1}=g_{k}-i j$ with $i j \in g_{k}$ and $i, j \in N^{G}$ for $k \geq 1$, and ending at $g_{K}=g^{N} \backslash g^{N^{G}}=g_{\text {assi,blue, all the }}$ green individuals have myopic incentives to delete their links with other green individuals. Indeed, consider a sequence starting at $g_{1}=g^{N}$, followed by $g_{k+1}=g_{k}-i j$ with $i \in N^{G}, j \in N_{i}\left(g_{k}\right) \cap N^{G}$, for $k=1, \ldots n^{G}-1$. Along this sequence, a green individual $i$ successively deletes all her links with the other green individuals and she has incentives to cut her $k$ th link to some green individual if and only if

$$
C>\Delta \frac{(n-2)(n-1-k)(n-2-k)}{n^{B}\left(n^{B}-1\right)^{2}} .
$$

This condition is satisfied since $C>\bar{C}_{2}$ and

$$
\bar{C}_{2}=\Delta \frac{(n-2)^{2}(n-3)}{n^{B}\left(n^{B}-1\right)^{2}} \geq \Delta \frac{(n-2)(n-1-k)(n-2-k)}{n^{B}\left(n^{B}-1\right)^{2}} .
$$

Farsighted blue individuals obtain their highest possible payoff in $g_{\text {assi,blue }}$ and myopic green individuals have no incentive to delete any link nor to add a new link since $C>\bar{C}_{2}$ and $c<\delta-\delta^{2}$. Hence, $\phi\left(g_{\text {assi,blue }}\right)=\emptyset$. So, since $\phi(g) \cap\left\{g_{\text {assi,blue }}\right\} \neq \emptyset$ for all $g \neq g_{\text {assi,blue }}$ and $\phi\left(g_{\text {assi,blue }}\right)=\emptyset$, the set $G=\left\{g_{\text {assi,blue }}\right\}$ is the unique myopic-farsighted stable set (any other set would violate (IS) and/or (ES)).

\section{References}

[1] Bisin, A., E. Patacchini, T. Verdier and Y. Zenou, 2016. Bend it like Beckham: Ethnic identity and integration. European Economic Review 90, 146-164.

[2] Bramoullé, Y., S. Currarini, M.O. Jackson, P. Pin and B.W. Rogers, 2012. Homophily and long-run integration in social networks. Journal of Economic Theory 147, 17541786 .

[3] Bramoullé, Y., A. Galeotti and B.W. Rogers, 2016. The Oxford Handbook of The Economics of Networks. Oxford University Press: Oxford, UK.

[4] Chwe, M.S., 1994. Farsighted coalitional stability. Journal of Economic Theory 63, 299-325.

[5] Currarini, S., M.O. Jackson and P. Pin, 2009. An economic model of friendship: Homophily, minorities, and segregation. Econometrica 77, 1003-1045. 
[6] de Marti, J. and Y. Zenou, 2017. Segregation in friendship networks. Scandinavian Journal of Economics 119, 656-708.

[7] De Palo, D., R. Faini and A. Venturini, 2007. The social assimilation of immigrants. Social Protection Discussion Paper SP 701. Washington, DC: World Bank.

[8] Dutta, B., S. Ghosal and D. Ray, 2005. Farsighted network formation. Journal of Economic Theory 122, 143-164.

[9] Dutta, B. and R. Vohra, 2017. Rational expectations and farsighted stability. Theoretical Economics 12, 1191-1227.

[10] Facchini, G., E. Patacchini and M.F. Steinhardt, 2015. Migration, friendship ties, and cultural assimilation. Scandinavian Journal of Economics 117, 619-649.

[11] Goyal, S., 2007. Connections: an introduction to the economics of networks. Princeton University Press: Princeton, NJ, USA.

[12] Herings, P.J.J., A. Mauleon and V. Vannetelbosch, 2009. Farsightedly stable networks. Games and Economic Behavior 67, 526-541.

[13] Herings, P.J.J., A. Mauleon and V. Vannetelbosch, 2017. Stable sets in matching problems with coalitional sovereignty and path dominance. Journal of Mathematical Economics 71, 14-19.

[14] Herings, P.J.J., A. Mauleon and V. Vannetelbosch, 2019. Stability of networks under horizon- $K$ farsightedness. Economic Theory 68, 177-201.

[15] Herings, P.J.J., A. Mauleon and V. Vannetelbosch, 2020. Matching with myopic and farsighted players. Working paper, UCLouvain.

[16] Jackson, M.O., 2008. Social and economic networks. Princeton University Press: Princeton, NJ, USA.

[17] Jackson, M. O. and B.W. Rogers, 2005. The economics of small worlds. Journal of the European Economic Association 3, 617-627.

[18] Jackson, M.O. and A. Watts, 2002. The evolution of social and economic networks. Journal of Economic Theory 106, 265-295.

[19] Jackson, M.O. and A. Wolinsky, 1996. A strategic model of social and economic networks. Journal of Economic Theory 71, 44-74.

[20] Johnson, C. and R.P. Gilles, 2000. Spatial social networks. Review of Economic Design 5, 273-299. 
[21] Kirchsteiger, G., M. Mantovani, A. Mauleon and V. Vannetelbosch, 2016. Limited farsightedness in network formation. Journal of Economic Behavior and Organization $128,97-120$.

[22] Luo, C., A. Mauleon and V. Vannetelbosch, 2020. Network formation with myopic and farsighted players. Working paper, UCLouvain.

[23] Mauersberger, F. and R. Nagel, 2018. Levels of reasoning in Keynesian beauty contests: A generative framework. In Handbook of Computational Economics vol.4 (C. Hommes and B. LeBaron, eds.), pp. 541-634, Elsevier, The Netherlands.

[24] Mauleon, A., J.J. Sempere-Monerris and V. Vannetelbosch, 2020. R\&D network formation with myopic and farsighted firms. Working paper, UCLouvain.

[25] Mauleon, A. and V. Vannetelbosch, 2016. Network formation games. In The Oxford Handbook of The Economics of Networks (Y. Bramoullé, A. Galeotti and B.W. Rogers, eds.), pp. 167-190, Oxford University Press, UK.

[26] Mauleon, A., V. Vannetelbosch and W. Vergote, 2011. von Neumann Morgernstern farsightedly stable sets in two-sided matching. Theoretical Economics 6, 499-521.

[27] Mele, A., 2017. A structural model of dense network formation. Econometrica 85(3), $825-850$

[28] Page, F.H., Jr. and M. Wooders, 2009. Strategic basins of attraction, the path dominance core, and network formation games. Games and Economic Behavior 66, 462487.

[29] Page, F.H., Jr., M. Wooders and S. Kamat, 2005. Networks and farsighted stability. Journal of Economic Theory 120, 257-269.

[30] Patacchini, E. and Y. Zenou, 2016. Racial identity and education in social networks. Social Networks 44, 85-94.

[31] Ray, D. and R. Vohra, 2015. The farsighted stable set. Econometrica 83(3), 977-1011.

[32] Ray, D. and R. Vohra, 2019. Maximality in the farsighted stable set. Econometrica 87(5), 1763-1779.

[33] Roketskiy, N., 2018. Competition and networks of collaboration. Theoretical Economics 13, 1077-1110.

[34] Teteryatnikova, M. and J. Tremewan, 2020. Myopic and farsighted stability in network formation games: an experimental study. Economic Theory, forthcoming.

[35] Verdier, T. and Y Zenou, 2017. The role of social networks in cultural assimilation. Journal of Urban Economics 97, 15-39. 